\begin{tabular}{|c|c|}
\hline Title & The Role of Dust in the Early Universe I : Protogal axy Evolution \\
\hline Author(s) & $\begin{array}{l}\text { Yamasawa, Daisuke; Habe, A sao; Kozasa, Takashi; Nozawa, Takaya; Hirashita, Hiroyuki; Umeda, Hidey uki; Nomoto, } \\
\text { Ken'ichi }\end{array}$ \\
\hline Citation & $\begin{array}{l}\text { The A strophysical Journal, } 735(1), 44 \\
\text { https://doi.org/10.1088/0004-637x /735/1/44 }\end{array}$ \\
\hline Issue Date & 2011-07-01 \\
\hline Doc URL & http:/hdl.handle.net/2115/53183 \\
\hline Rights & Published under licence in The A strophysical Journal by IOP Publishing Ltd. \\
\hline Type & article \\
\hline File Information & 0004-637X_735_1_44.pdf \\
\hline
\end{tabular}

Instructions for use 


\title{
THE ROLE OF DUST IN THE EARLY UNIVERSE. I. PROTOGALAXY EVOLUTION
}

\author{
Daisuke Yamasawa ${ }^{1}$, Asao Habe ${ }^{1}$, Takashi Kozasa ${ }^{1}$, Takaya Nozawa ${ }^{2}$, Hiroyuki Hirashita ${ }^{3}$, \\ HidEYUKI UMEDA ${ }^{4}$, AND KEN'ICHI NOMOTO ${ }^{2}$ \\ ${ }^{1}$ Department of Cosmosciences, Graduate School of Science, Hokkaido University, Sapporo, Hokkaido 060-0810, Japan; yamasawa@ astro1.sci.hokudai.ac.jp \\ ${ }^{2}$ Institute for the Physics and Mathematics of the Universe, University of Tokyo, Kashiwa, Chiba 277-8583, Japan \\ ${ }^{3}$ Institute of Astronomy and Astrophysics, Academia Sinica, P.O. Box 23-141, Taipei 10617, Taiwan \\ ${ }^{4}$ Department of Astronomy, School of Science, University of Tokyo, Hongo, Tokyo 113-0033, Japan \\ Received 2010 November 29; accepted 2011 March 30; published 2011 June 14
}

\begin{abstract}
We develop one-zone galaxy formation models in the early universe, taking into account dust formation and evolution by supernova (SN) explosions. We focus on the time evolution of dust size distribution, because $\mathrm{H}_{2}$ formation on the dust surface plays a critical role in the star formation process in the early universe. In the model, we assume that star formation rate (SFR) is proportional to the total amount of $\mathrm{H}_{2}$. We consistently treat (1) the formation and size evolution of dust, (2) the chemical reaction networks including $\mathrm{H}_{2}$ formation both on the surface of dust and in gas phase, and (3) the SFR in the model. First, we find that, because of dust destruction due to both reverse and forward shocks driven by $\mathrm{SNe}, \mathrm{H}_{2}$ formation is more suppressed than in situations without such dust destruction. At the galaxy age of $\sim 0.8 \mathrm{Gyr}$, for galaxy models with virial mass $M_{\mathrm{vir}}=10^{9} M_{\odot}$ and formation redshift $z_{\text {vir }}=10$, the molecular fraction is 2.5 orders of magnitude less in the model with dust destruction by both shocks than that in the model without dust destruction. Second, we show that the $\mathrm{H}_{2}$ formation rate strongly depends on the interstellar medium (ISM) density around SN progenitors. The SFR in higher ISM density is lower, since dust destruction by reverse shocks is more effective in higher ISM density. We conclude that not only the amount but also the size distribution of dust related to star formation activity strongly affects the evolution of galaxies in the early universe.
\end{abstract}

Key words: dust, extinction - early universe - galaxies: evolution - galaxies: formation - galaxies: ISM

Online-only material: color figures

\section{INTRODUCTION}

Understanding galaxy evolution in the early universe remains one of the most important goals of modern astrophysics. Modeling of primeval galaxy formation requires an accurate treatment of the star formation process in low-metallicity gas (Jappsen et al. 2007; Glover \& Jappsen 2007; Smith et al. 2008, 2009; Jappsen et al. 2009b, 2009a). A critical challenge for achieving this goal is our poor understanding of how gas is converted into stars under different conditions (Krumholz \& McKee 2005; Robertson \& Kravtsov 2008; Gnedin et al. 2009). In particular, star formation efficiency in the primeval galaxy is still uncertain.

The standard approach in theoretical studies of galaxy formation so far is to adopt a recipe that ties the star formation rate (SFR) to gas density both in semi-analytic models (e.g., Cole et al. 2000) and in numerical simulations (e.g., Springel et al. 2005). Such a recipe is based on the empirical correlations observed in local galaxies, namely, the Kennicutt-Schmidt law (Kennicutt 1998). These correlations have only been studied relatively well for nearby massive or star bursting galaxies. However, for galaxies with low surface brightness and/or low metallicity, this empirical relation may not be valid. Indeed, both nearby metal-poor galaxies (Bigiel et al. 2008) and high-redshift galaxies (Wolfe \& Chen 2006) provide a variety of clues suggesting that gas conversion into stars in low-mass, low-metallicity galaxies is very inefficient.

The star formation efficiency may depend on the ability to convert a fraction of gas mass into molecular form. Molecular hydrogen is produced by chemical reactions in the gas phase in first galaxy halos. In the reionization era, $\mathrm{H}_{2}$ molecule disso- ciation by the Lyman-Werner ultraviolet (UV) background between 11.2 and $13.6 \mathrm{eV}$ is important in the lower mass $\mathrm{H}_{2}$ cooling halos. Gas condensation in the lower mass $\mathrm{H}_{2}$ cooling halos can be delayed by the Lyman-Werner background (Machacek et al. 2001, 2003; Yoshida et al. 2003; Susa 2007; Wise \& Abel 2007; O'Shea \& Norman 2008). The Lyman-Werner background thus increases cooling times in the centers of such halos. As a result, the minimum mass of a star-forming halo increases with Lyman-Werner background intensity. The Lyman-Werner background becomes less of an issue in atomic line cooling halos as Ly $\alpha$ cooling provides ample amounts of free electrons for $\mathrm{H}_{2}$ cooling, and they become self-shielding to this radiation (O'Shea \& Norman 2008; Susa 2008; Wise \& Abel 2008; Wise \& Cen 2009).

In the later epoch, dust ejected by stars in galaxies effectively shields the Lyman-Werner background and acts as an effective catalyst for $\mathrm{H}_{2}$ molecule production on the dust grains. In simulations with star formation models based on molecular hydrogen (Robertson \& Kravtsov 2008; Gnedin et al. 2009), once the gas is enriched up to $Z \sim 0.01-0.1 Z_{\odot}$, the subsequent star formation and enrichment of metal and dust is greatly accelerated. Gnedin et al. (2009) show that the transition from atomic to molecular hydrogen depends primarily on metallicity, assuming that the dust abundance is directly related to metallicity.

Dust plays a crucial role in star formation: (1) molecular hydrogen is produced more efficiently on dust grains than in the gas phase, (2) dust shields dissociate UV radiation, and (3) dust allows the formation of low-mass stars in low-metallicity environments and hence affects the initial mass function (IMF; Omukai et al. 2005; Schneider et al. 2006; Schneider \& Omukai 2010; Omukai et al. 2010). 
In theoretical studies on the molecular abundance in the interstellar medium (ISM), dust abundance is often scaled with the metallicity, and dust grain properties are assumed to be the same as in the local ISM. However, the composition of dust is likely to be different in early galaxies. The observational evidence is that the dust extinction curves of the broad absorption line quasars at $z>4$ are likely to be due to the Type II supernova (SN II) dust (Maiolino et al. 2004; Gallerani et al. 2010).

Since the lifetime of an SN II progenitor is short, SN II can be the dominant production source of dust grains in young $(<1 \mathrm{Gyr})$ galaxies. Primeval SNe produced by Population III stars (Bromm et al. 2003; Kitayama \& Yoshida 2005; Whalen et al. 2008) may contribute to dust production (Nozawa et al. 2003; Schneider et al. 2004). The winds of evolved lowmass stars contribute considerably to dust formation in nearby galaxies, but the cosmic time is not long enough for such stars to evolve at high redshift $(z>5)$, where all galaxies should have ages younger than $\simeq 1$ Gyr. Contribution of dust production by low-mass stars is not dominant in such young galaxies. In addition, dust is destroyed by SN shocks. Thus, the modeling of dust evolution in galaxies requires an accurate treatment of production and destruction of dust grains together with star formation activities (Hirashita \& Ferrara 2002).

In this paper, we investigate not only the evolution of dust mass but also the time evolution of dust size distribution. The dust size distribution evolves rapidly because of the destruction by sputtering in the high-velocity shocks driven by $\mathrm{SNe}$. Collision of the expanding SN ejecta with the surrounding ISM creates a forward shock at the interface between the ejecta and the ISM (Nozawa et al. 2006), and a reverse shock that penetrates into the ejecta (Bianchi \& Schneider 2007; Nozawa et al. 2007; Nath et al. 2008; Silvia et al. 2010). Since the erosion rate by sputtering does not strongly depend on the grain size, small grains are predominantly destroyed regardless of grain species. Therefore, the fraction of small-size grains relatively decreases with galaxy evolution.

We focus on the effects of molecular hydrogen abundance on the SFR in the early stage of galaxy evolution, taking into account molecular formation on dust, since $\mathrm{H}_{2}$ formation on the dust surface is very effective (Hirashita \& Ferrara 2002; Cazaux \& Spaans 2004). Hirashita \& Ferrara (2002) show that this effect causes an enhancement of the SFR by an order of magnitude on a timescale of 3-5 galactic dynamical time. However, they assumed a single dust grain size $(\sim 0.03 \mu \mathrm{m})$. We adopt more accurate analytic formulae for the formation of molecular hydrogen on dust grains than those in Hirashita \& Ferrara (2002) by using the results of dust size distribution by Nozawa et al. (2006, 2007).

This is the first study of galaxy evolution considering dust size evolution for halo masses above $10^{8}-10^{9}$ in the high redshift $(5<z<10)$, whose interiors we expect to be roughly selfshielded from both ionizing and Lyman-Werner UV radiation. To clearly show the dependence of galaxy properties on dust destruction, we use a simple one-zone galaxy model.

The paper is organized as follows: In Section 2, we describe the dust evolution model. In Section 3, we explain our onezone galaxy model. In Section 4, we present the results. In Section 5, we discuss the effects of the dust size evolution on the $\mathrm{H}_{2}$ formation process and conclude by summarizing our results. Throughout this paper, we adopt the cosmological parameters from the third-year Wilkinson Microwave Anisotropy Probe results (Spergel et al. 2007): $\Omega_{\Lambda}=0.76, \Omega_{M}=0.24$, $\Omega_{b}=0.04$, and $H_{0}=73 \mathrm{~km} \mathrm{~s}^{-1} \mathrm{Mpc}^{-1}$.

\section{DUST EVOLUTION MODEL}

\subsection{Source of Dust in the Early Universe}

SNe II are believed to be the dominant sources of dust at a high redshift of $z>5$ because of the short lifetimes $\left(<10^{7} \mathrm{yr}\right)$ of their massive progenitors (e.g., Dwek et al. 2007; Gallerani et al. 2010; Gall et al. 2011). Dust formation in the ejecta of primordial SNe II has been investigated theoretically (Todini \& Ferrara 2001; Nozawa et al. 2003; Cherchneff \& Dwek 2010). The amount and the size distributions of dust grains injected into the ISM have been investigated by considering the destruction in SN remnants (SNRs; Bianchi \& Schneider 2007; Nozawa et al. 2007; Nath et al. 2008; Silvia et al. 2010). Although the amount of dust that forms in the ejecta is still debated (Kozasa et al. 2009, for review), the recent observations of Cas-A SNR revealed the presence of $\sim 0.07 M_{\odot}$ dust condensed in the ejecta (Barlow et al. 2010; Sibthorpe et al. 2010), which is consistent with the dust mass predicted by the theoretical model taking into account formation and destruction processes of dust in an SN IIb (Nozawa et al. 2010). Valiante et al. (2009) and Dwek \& Cherchneff (2011) have proposed that the contribution from asymptotic giant branch (AGB) stars in the high-redshift quasar $\mathrm{J} 1148+5251$ cannot be neglected for the total dust budget even at $z \sim 6$. However, the size distribution of dust formed in the mass-loss wind of AGB stars has not yet been fully studied (Ferrarotti \& Gail 2006; Zhukovska et al. 2008). If SNe Ia could occur in such an early epoch, they are unlikely to be efficient sources of dust (Nozawa et al. 2011). Therefore, in order to follow the evolution of dust size distribution and reveal the resulting influence on galaxy evolution, we consider SNe II as the source of dust in the early universe.

The basic quantity for governing the production and destruction history of dust by SNe II is the rate of SN II explosions, $\gamma_{\mathrm{SN}}(t)$, given by

$$
\gamma_{\mathrm{SN}}(t)=\int_{m_{\mathrm{SN}}^{l}}^{m_{\mathrm{SN}}^{u}} d m \Psi(t-\tau(m)) \phi(m),
$$

where $\Psi(t)$ is the SFR at time $t, \phi(m)$ is the stellar IMF, $\tau(m)$ is the lifetime of a star whose mass is $m$, and $m_{\mathrm{SN}}^{u}$ and $m_{\mathrm{SN}}^{l}$ are the upper and lower mass limits of SN II progenitors, respectively. In this paper we adopt the Salpeter IMF $\left(\phi(m) \propto m^{-2.35}\right.$; Salpeter $1955)$ with the stellar mass range between $0.1 M_{\odot}$ and $60 M_{\odot}$; we assume $m_{\mathrm{SN}}^{l}=8 M_{\odot}$ and $m_{\mathrm{SN}}^{u}=40 M_{\odot}$ (Heger et al. 2003). For $\tau(m)$, we adopt the model of zero-metallicity stars without mass loss (Schaerer 2002).

In this paper, we do not consider Population III stars, for simplicity. In the forthcoming paper, we will consider the possible contribution of Population III stars. Population III stars formed out of the primordial gas are considered to be much more massive than Population I/II stars (Yoshida et al. 2008; Bromm et al. 2009 for reviews); thus, the primordial IMF might be biased toward a higher mass $\left(\gtrsim 10 M_{\odot}\right)$ than that in the present universe. Furthermore, Population III stars as massive as $140-260 M_{\odot}$ are predicted to end their lives as pair-instability SNe (PISNe; Heger \& Woosley 2002) and to produce a large amount of metals and dust (Nozawa et al. 2003; Schneider et al. 2004). However, Joggerst et al. (2010a, 2010 b) address the growing nucleosynthetic "forensic" evidence that the majority of primordial stars may have been $15-40 M_{\odot}$ objects. On the other hand, once the gas is enriched up to a critical metallicity of $Z \simeq 10^{-6}-10^{-5} Z_{\odot}$, formation of lowmass stars is triggered, leading to the transition of the star 


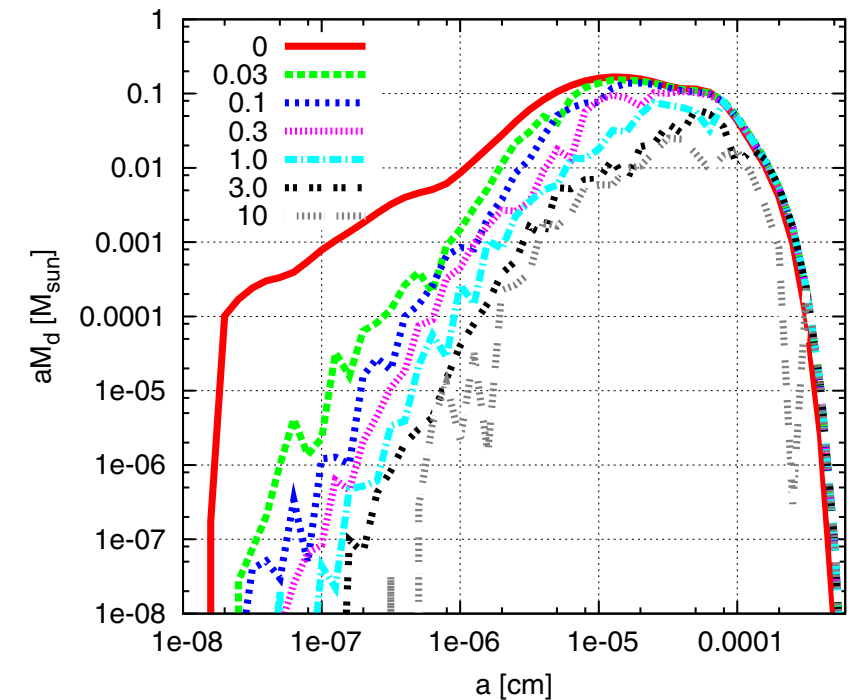

Figure 1. IMF-averaged mass distributions of dust formed in the ejecta and injected into ISM per SN II: the solid line denotes the mass distribution of dust before the destruction through a reverse shock, $a \overline{\mathcal{M}_{d}^{0}}(a)$; the dashed lines and the dotted lines denote the mass distributions of dust at the injection into ISM after the destruction through a reverse shock, $a \overline{\mathcal{M}}_{d}^{n_{\mathrm{SN}}}(a)$, for the number densities of gas around an SN progenitor, $n_{\mathrm{SN}}=0.03,0.1,0.3,1.0,3.0$, and $10.0 \mathrm{~cm}^{-3}$, which are used to annotate the curves. The horizontal axis shows the radius of dust in units of $\mathrm{cm}$. The vertical axis is the mass distribution of dust $a \overline{\mathcal{M}_{d}^{n_{\mathrm{SN}}}}(a)$ in units of $M_{\odot}$.

(A color version of this figure is available in the online journal.)

formation mode from massive Population III stars to low-mass Population I/II stars, if dust is present (Omukai et al. 2005; Schneider et al. 2006; Schneider \& Omukai 2010; Omukai et al. 2010). If there is no dust, the transition of the star formation mode is expected to occur at $10^{-3.5} Z_{\odot}$ (Mackey et al. 2003). In this case, the cosmic microwave background (CMB) limits the lower masses of stars to a few tens of $M_{\odot}$ (Smith et al. 2008; Schneider \& Omukai 2010). Formation history of galaxies considering the time-dependent IMF from the top-heavy to the Salpeter-like IMF and taking into account the production and destruction of dust by PISNe will be explored in the forthcoming paper (D. Yamasawa et al. 2011, in preparation).

\subsection{Dust Injected from SNe II into ISM}

Throughout this paper, we adopt the models by Nozawa et al. $(2003,2007)$ for dust formation and destruction. Nozawa et al. (2003) investigated the dust production in the ejecta of primordial SNe II as well as PISNe, applying a theory of nonsteady-state nucleation and grain growth. They revealed the grain species formed in the ejecta and their size distributions for the unmixed and mixed elemental compositions within the He core. In what follows, we apply the results of calculation for the unmixed ejecta of SNe II with the progenitor mass $m=13,20,25$, and $30 M_{\odot}$ and the explosion energy $10^{51} \mathrm{erg}$, and extrapolate the data to the mass range from 8 to $40 M_{\odot}$.

The solid line in Figure 1 shows the IMF-averaged mass distribution of dust formed in the ejecta of SNe II, $\overline{\mathcal{M}_{d}^{0}}(a)$, which is weighted by the Salpeter IMF and is summed up over all the grain species as

$$
\overline{\mathcal{M}_{d}^{0}}(a)=\sum_{j} \overline{\mathcal{M}_{d, j}^{0}}(a)=\frac{\sum_{j} \int_{\mathrm{m}_{\mathrm{SN}}^{l}}^{m_{\mathrm{SN}}^{u}} d m \mathcal{M}_{d, j}^{0}(a, m) \phi(m)}{\sum_{j} \int_{m_{\mathrm{SN}}^{l}}^{m_{\mathrm{SN}}^{u}} d m \phi(m)},
$$

where $\mathcal{M}_{d, j}^{0}(a, m) d a$ is the mass of the $j$ th dust species produced in an SN II with radii between $a$ and $a+d a$ as a function of progenitor mass $m$, and superscript 0 indicates the case with no destruction by a reverse shock. In Figure 1, we plot $a \overline{\mathcal{M}_{d}^{0}}(a)$ in the vertical axis to make clear the mass fraction in each logarithmic bin. We can see that the grain radii range from a few $\AA$ up to a few $\mu \mathrm{m}$ and that the size spectrum of dust in mass has a peak at $a \sim 0.1 \mu \mathrm{m}$.

In the course of their injection into ISM, dust grains formed in the ejecta are destroyed due to sputtering in the hot gas between the reverse and forward shocks, which is hereafter referred to as destruction by the reverse shock. Nozawa et al. (2007) investigated the survival of the newly formed dust in the shocked gas within the SNRs expanding into the uniform ISM with hydrogen number densities of $n_{\mathrm{SN}}=0.1,1.0$, and $10.0 \mathrm{~cm}^{-3}$ and showed that the destruction efficiency of newly formed dust is not only sensitive to the initial size distribution but also strongly depends on $n_{\mathrm{SN}}$. To investigate the dependence of destruction of dust on the ISM densities, we extend their models to six cases of $n_{\mathrm{SN}}=0.03,0.1,0.3,1.0,3.0$, and $10.0 \mathrm{~cm}^{-3}$.

Figure 1 shows the IMF-averaged mass distribution of dust, $\overline{\mathcal{M}_{d}^{n_{\mathrm{SN}}}}(a)$, injected into the ISM after destruction by the reverse shock for $n_{\mathrm{SN}}=0.03,0.1,0.3,1.0,3.0$, and $10.0 \mathrm{~cm}^{-3}$, which is weighted by the Salpeter IMF and is summed up over all the grain species as in Equation (2). The mass distribution of the $j$ th dust species for the case of $n_{\mathrm{SN}}, \overline{\mathcal{M}_{d, j}^{n_{\mathrm{SN}}}}(a)$, is the IMF-averaged one after the destruction by the reverse shock. We can see that the change in the dust mass distribution through processing in SNRs becomes more (less) prominent for higher (lower) gas density; small-size grains get deficient with increasing $n_{\mathrm{SN}}$. The dust grains with radii below $0.01 \mu \mathrm{m}$ are preferentially destroyed by sputtering for $n_{\mathrm{SN}}>0.03 \mathrm{~cm}^{-3}$, while dust with radii larger than $\sim 1 \mu \mathrm{m}$ is almost intact for $n_{\mathrm{SN}} \leqslant 3.0 \mathrm{~cm}^{-3}$. As a result, the mass of dust injected into the ISM is dominated by grains with radii above $\sim 0.1 \mu \mathrm{m}$.

The total geometrical cross section of dust per metal mass is an important quantity for $\mathrm{H}_{2}$ formation on the grain surface at a certain metallicity level. The total geometrical cross section of dust injected into the ISM from an SN depends on the gas density around the $\mathrm{SN}$ progenitor, $n_{\mathrm{SN}}$. In many papers, the total geometrical cross section of dust is scaled to metal mass under the following two assumptions: (1) the depletion factor, which is defined as the dust mass per metal mass, is identical to that in the Milky Way (MW); and (2) the dust size distribution, which determines dust area per unit dust volume $\left\langle a^{2}\right\rangle /\left\langle a^{3}\right\rangle$, is the same as that in the MW. However, the depletion factor and the size distribution of the SN II dust are quite different from those in the MW.

Figure 2 shows the depletion factor, $\overline{M_{\mathrm{SN}, \mathrm{d}}^{n_{\mathrm{SN}}}} / \overline{m_{m}}$, after the destruction by the reverse shock as a function of ISM density $n_{\mathrm{SN}}$, where $M_{\mathrm{SN}, \mathrm{d}}^{n_{\mathrm{SN}}}$ is the IMF-averaged total dust mass ejected into the ISM by an SN,

$$
\overline{M_{\mathrm{SN}, \mathrm{d}}^{n_{\mathrm{SN}}}}=\int_{0}^{\infty} d a \overline{\mathcal{M}_{d}^{n_{\mathrm{SN}}}}(a)
$$

and $\overline{m_{m}}$ is the IMF-averaged total metal mass ejected into the ISM,

$$
\overline{m_{m}}=\sum_{i} \overline{m_{\mathrm{m}, i}}=\frac{\sum_{i} \int_{m_{\mathrm{SN}}^{l}}^{m_{\mathrm{SN}}^{u}} m_{\mathrm{m}, i}(m) \phi(m) d m}{\sum_{i} \int_{m_{\mathrm{SN}}^{l}}^{m_{\mathrm{SN}}^{l}} \phi(m) d m} .
$$




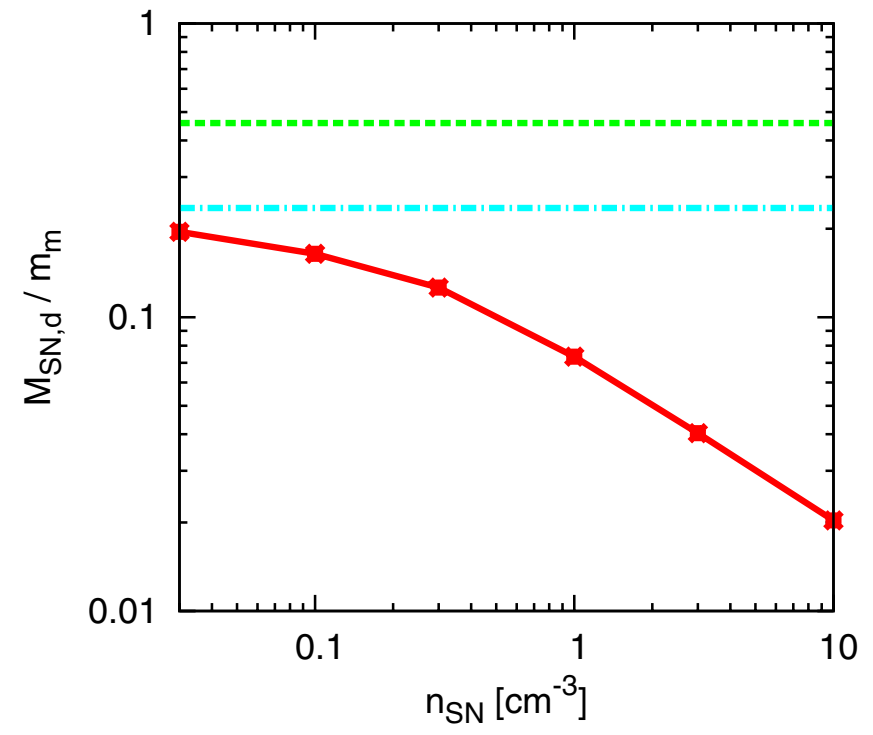

Figure 2. Depletion factor, $\overline{M_{\mathrm{SN}, \mathrm{d}}^{n_{\mathrm{SN}}}} / \overline{m_{m}}$, for various dust destruction models with the densities of ISM around the SN II progenitor, $n_{\mathrm{SN}}=$ $0.03,0.1,0.3,1,3$, and $10 \mathrm{~cm}^{-3}$. The dot-dashed line represents the depletion factor, $\overline{M_{\mathrm{SN}, \mathrm{d}}^{0}} / \overline{m_{m}}$, for the model without reverse shock destruction. The ejected metal mass per SN II is taken from Umeda \& Nomoto (2002). The dotted line represents the typical ratio in MW, where we assume the dust-to-gas mass ratio to be $\mathcal{D}=0.00934$ (Pollack et al. 1994) and the ratio of metal mass to hydrogen mass to be 0.0204 (Omukai 2000).

(A color version of this figure is available in the online journal.)

Here, $m_{\mathrm{m}, i}(m)$ is the mass of the $i$ th element of metal ejected from an SN with progenitor $m$, taken from Umeda \& Nomoto (2002). The depletion factor in the case of $n_{\mathrm{SN}}=1.0 \mathrm{~cm}^{-3}$ is a factor of six smaller than that in the MW.

Figure 3 shows the ratio of the total geometric cross section of dust to the total metal mass, $\overline{D_{\text {area }}^{n_{\mathrm{SN}}}} / \overline{m_{\mathrm{m}}}$, after the destruction by the reverse shock as a function of ISM density $n_{\mathrm{SN}}$. The total geometrical cross section of dust weighted by the Salpeter IMF, $\overline{D_{\text {area }}^{n_{\mathrm{SN}}}}$, is written as

$$
\overline{D_{\text {area }}^{n_{\mathrm{SN}}}}=\sum_{j} \frac{3}{4 \rho_{j}} \int_{0}^{\infty} d a \overline{\mathcal{M}_{d, j}^{n_{\mathrm{SN}}}}(a) / a
$$

where $\rho_{j}$ is the bulk density of the $j$ th dust species. The ratio, $\overline{D_{\text {area }}^{n_{\mathrm{SN}}}} / \overline{m_{\mathrm{m}}}$, is smaller for larger $n_{\mathrm{SN}}$ because small dust grains are efficiently destroyed by sputtering in the SNR under large $n_{\mathrm{SN}}$; note that the surface area per dust mass is larger for smaller size grains. Also with increasing $n_{\mathrm{SN}}$, the reverse shock becomes stronger and destroys dust by sputtering more effectively (see Nozawa et al. 2007). In addition, we plot the ratio for the case without reverse shock together with the typical value in the MW for comparison. $\overline{D_{\text {area }}^{n_{\mathrm{SN}}}} / \overline{m_{\mathrm{m}}}$ in the model without reverse shock is a factor of four smaller than that in the MW. $\overline{D_{\text {area }}^{n_{\mathrm{SN}}}} / \overline{m_{\mathrm{m}}}$ in the case with $n_{\mathrm{SN}}=1.0 \mathrm{~cm}^{-3}$ is 40 times smaller than that in the MW. Thus, the rescaling of the cross section of dust by the metal mass using the MW value results in significant overestimate for $\mathrm{H}_{2}$ formation in high-redshift galaxies.

\subsection{Destruction of Interstellar Dust by SN Forward Shocks}

Dust grains injected into the ISM are subjected to destruction by the blast waves (the high-velocity interstellar shocks) driven by the ambient SNe (e.g., Jones et al. 1994). Nozawa et al. (2006)

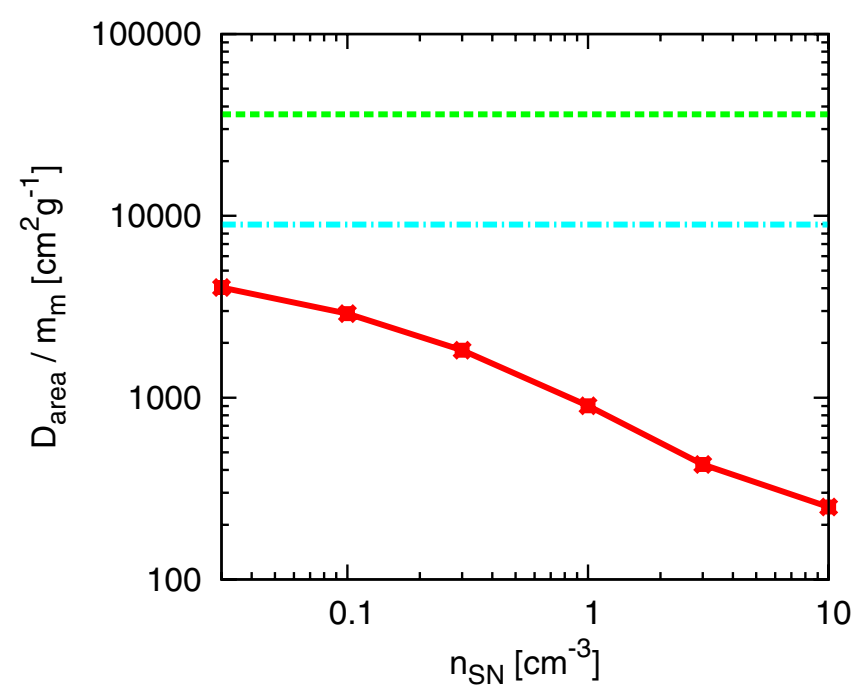

Figure 3. Ratio of total dust cross section to total metal mass, $\overline{D_{\text {area }}^{n_{\mathrm{SN}}}} / \overline{m_{\mathrm{m}}}$, for various dust destruction models with the densities of ISM around the SN II progenitor, $n_{\mathrm{SN}}=0.03,0.1,0.3,1,3$, and $10 \mathrm{~cm}^{-3}$. The dot-dashed line represents the ratio, $\overline{D_{\text {area }}^{0}} / \overline{m_{\mathrm{m}}}$, for the model without reverse shock destruction. The ejected metal mass per SN II is taken from Umeda \& Nomoto (2002). The dotted line represents the ratio in our galaxy, where we assume a dust property as a size distribution, $f(a) \propto a^{-3.5}\left(5 \times 10^{-7} \mathrm{~cm}<a<2.5 \times 10^{-5} \mathrm{~cm}\right)$ (Mathis et al. 1977; Draine \& Lee 1984) and a dust-to-gas mass ratio, $\mathcal{D}=0.00934$ (Pollack et al. 1994), and that the ratio of metal mass to hydrogen mass is 0.0204 and the bulk density of dust is $3.0 \mathrm{~g} \mathrm{~cm}^{-3}$ (Omukai 2000).

(A color version of this figure is available in the online journal.)

investigated the processing of interstellar dust by sputtering in the hot gas swept up by the SN forward shock. Adopting the dust model by Nozawa et al. (2003) as the size distribution of interstellar dust, Nozawa et al. (2006) have shown that the destruction efficiency of dust depends on the ISM density and the explosion energy of $\mathrm{SNe}$ as well as the initial size distribution of dust. It should be noted that the size distribution and the destruction efficiency change as functions of time because interstellar dust is supplied from $\mathrm{SNe}$ and processed in ISM successively according to star formation activity. Thus, we must deal with the destruction process in a way that is applicable to any dust size distribution to explore the global evolution of dust size distribution.

In order to evaluate the destruction efficiency of interstellar dust for any initial size distribution, here we introduce the conversion efficiency as defined below. Consider that the $j$ th dust species residing in the ISM, whose size distribution is given by the number of dust grains with radii between $a$ and $a+d a$, $f_{j}(a) d a$, is processed by sputtering in hot plasma produced through a single passage of an $\mathrm{SN}$ shock. The conversion efficiency $\eta_{j}\left(a, a^{\prime}\right)$ is defined as the number fraction of dust grains with radii between $a^{\prime}$ and $a^{\prime}+d a^{\prime}$ that are converted to grains with radii between $a$ and $a+d a$ by sputtering through the passage of an SN shock. The number of dust grains with radii between $a$ and $a+d a$ produced by the sputtering is given as $\eta_{j}\left(a, a^{\prime}\right) f_{j}\left(a^{\prime}\right) d a^{\prime}$. Note that $\eta_{j}\left(a, a^{\prime}\right)=0$ for $a>a^{\prime}$. Then the change in the number of dust grains with radii between $a$ and $a+d a$ caused by shock processing is given by

$$
\begin{aligned}
d N_{j}(a) & =\sum_{a^{\prime}>a}^{\infty} \eta_{j}\left(a, a^{\prime}\right) f_{j}\left(a^{\prime}\right) d a^{\prime}-\left[1-\eta_{j}(a, a)\right] f_{j}(a) d a \\
& =\int_{0}^{\infty} \eta_{j}\left(a, a^{\prime}\right) f_{j}\left(a^{\prime}\right) d a^{\prime}-f_{j}(a) d a
\end{aligned}
$$




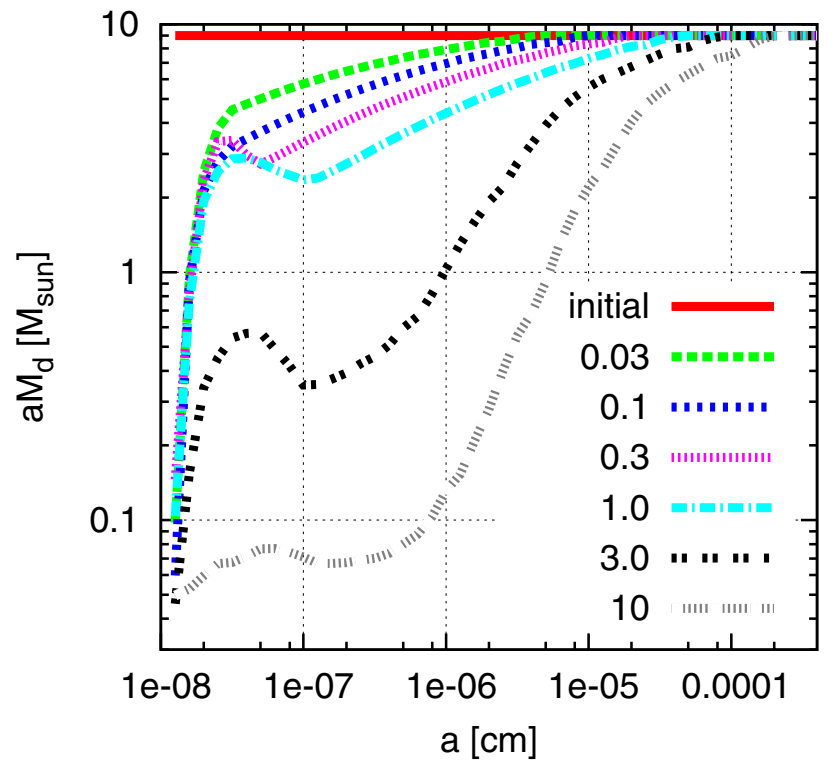

Figure 4. Dust mass, $\sum_{j} a \mathcal{M}_{d, j}(a)$, processed by a forward shock driven by an SN II explosion in the ambient ISM gas density of $n_{\mathrm{SN}}=0.03,0.1,0.3,1.0$, 3.0 , and $10.0 \mathrm{~cm}^{-3}$, which are used to annotate the curves, and metallicity of $Z=10^{-4} Z_{\odot}$. We assume the initial size distribution to be $a \mathcal{M}_{d, j}(a)=1$ for each species $j$. In the calculations, we use the conversion efficiency $\eta_{j}\left(a, a^{\prime}\right)$ evaluated for SN explosion energy, $10^{51} \mathrm{erg}$, and progenitor mass, $m=20 M_{\odot}$. (A color version of this figure is available in the online journal.)

The corresponding change of the mass is given by

$$
d M_{d, j}(a)=\frac{4 \pi}{3} a^{3} \rho_{j} \int_{0}^{\infty} \eta_{j}\left(a, a^{\prime}\right) f_{j}\left(a^{\prime}\right) d a^{\prime}-\mathcal{M}_{d, j}(a) d a
$$

where $\mathcal{M}_{d, j}(a) d a$ is the mass of the pre-shocked dust. The size distribution function after the shock processing $f_{j}^{\prime}(a)$ is given by $f_{j}^{\prime}(a)=f_{j}(a)+d N_{j} / d a$.

The conversion efficiency $\eta\left(a, a^{\prime}\right)$ and the mass of ISM gas swept up by shock $M_{\text {swept }}$ depend on the progenitor mass, expanding energy, and type of the $\mathrm{SN}$ as well as the structure, number density, and metallicity of the ISM gas. For these parameters of SNe and ambient ISM, once $M_{\text {swept }}$ and $\eta\left(a, a^{\prime}\right)$ for each dust species are calculated, the time evolution of dust mass and size distribution can be followed in a consistent way with the star formation activity in galaxies as described in Section 3.

The calculations of $\eta\left(a, a^{\prime}\right)$ and $M_{\text {swept }}$ are performed using the method developed by Nozawa et al. (2006) as follows: the efficiency of dust destruction increases with increasing explosion energy and/or increasing $n_{\mathrm{SN}}$ but is almost independent of the progenitor mass as long as the explosion energy is the same (Nozawa et al. 2006). We assume that SNe driving high-velocity shock in ISM are represented by SN II with the progenitor mass of $20 M_{\odot}$ and the explosion energy of $10^{51} \mathrm{erg}$. The ISM surrounding the $\mathrm{SN}$ is considered to be uniform with hydrogen number densities $n_{\mathrm{SN}}=0.03,0.1,0.3,1.0,3.0$, and $10 \mathrm{~cm}^{-3}$. By distributing dust grains with radius $a^{\prime}$ uniformly in the ISM, the conversion efficiency $\eta\left(a, a^{\prime}\right)$ is evaluated for each grain species by calculating the erosion of dust by sputtering until the truncation time $t_{\mathrm{tr}}$, which is defined as a time when the shock velocity is decelerated below $100 \mathrm{~km} \mathrm{~s}^{-1}$ (see Nozawa et al. 2006 , for the details). In the calculations, the radii of grains in the ISM range from 0.00013 to $6.3 \mu \mathrm{m}$ for each grain species.

In Figure 4, we present the changes in the dust size distributions due to the shock processing for different ISM den- sities, where the initial mass distribution of dust is set to be $a \mathcal{M}_{d, j}(a)=1$ for clarity. As seen in the figure, small-size grains are destroyed due to the erosion by sputtering, and more dust grains are processed for a higher ISM density.

The mass of gas swept up by the forward shock until the truncation time $t_{\mathrm{tr}}, M_{\text {swept }}$, depends not only on the ISM density but also on the initial metallicity of the gas in the ISM. As the line cooling by heavy elements becomes more efficient for a higher gas metallicity, the forward shock is decelerated more quickly, resulting in a smaller $M_{\text {swept }}$. By fitting $M_{\text {swept }}$ calculated for different $n_{\mathrm{SN}}$ and $Z$, we derive the following approximation formula,

$$
M_{\text {swept }} / M_{\odot}=1535 n_{\mathrm{SN}}^{-0.202}\left[\left(Z / Z_{\odot}\right)+0.039\right]^{-0.298},
$$

whose fitting accuracy is within $16 \%$ for $0.03 \mathrm{~cm}^{-3} \leqslant n_{\mathrm{SN}} \leqslant$ $30 \mathrm{~cm}^{-3}$ and for $10^{-4} \leqslant Z / Z_{\odot} \leqslant 1.0$.

\subsection{Formulation of Dust Size Evolution}

In terms of the conversion efficiency describing the processing of dust by sputtering, here we formulate the time evolution of the mass of $j$ th dust grains with radii between $a$ and $a+\Delta a$ in our model galaxies, $\Delta M_{d, j}(a, t)=\frac{4 \pi}{3} a^{3} \rho_{j} f_{j}(a, t) \Delta a$, as

$$
\begin{aligned}
\frac{d \Delta M_{d, j}(a, t)}{d t}= & \overline{\Delta M_{\mathrm{SN}, d, j}}(a) \gamma_{\mathrm{SN}}(t) \\
& -\frac{M_{\mathrm{Swept}}}{M_{\mathrm{ISM}}(t)} \gamma_{\mathrm{SN}}(t) \times\left\{\Delta M_{d, j}(a, t)\right. \\
& \left.-\int_{0}^{\infty} d a^{\prime} \eta_{j}\left(a, a^{\prime}\right) f_{j}\left(a^{\prime}, t\right) \rho_{j} \frac{4 \pi}{3} a^{3}\right\} \\
& -\Psi(t) \frac{\Delta M_{d, j}(a, t)}{M_{\mathrm{ISM}}(t)}
\end{aligned}
$$

where $M_{\mathrm{ISM}}(t)$ is the total mass of gas and dust, and $f_{j}(a, t)$ is the size distribution function of dust species $j$ in the ISM at a time $t$. We note that $\left(M_{\text {swept }} \gamma_{\mathrm{SN}}(t) / M_{\mathrm{ISM}}(t)\right)^{-1}$ is a timescale of sweeping the whole ISM by SNe. The IMF-averaged mass of dust species $j$ with radii between $a$ and $a+\Delta a$ injected from SNe II into the ISM with number density $n_{\mathrm{ISM}, \mathrm{SN}}$ is defined by $\overline{\Delta M_{\mathrm{SN}, d, j}}(a)=\overline{\mathcal{M}_{d, j}^{n_{\mathrm{SN}}}}(a) \Delta a$. The first term on the right-hand side is the injection rate of dust from SNe II. The second term is the destruction rate of interstellar dust by SN blast waves, and the third term is the rate at which the interstellar dust is incorporated into stars.

\section{GALAXY EVOLUTION MODEL}

\subsection{Dark Matter Halo and Physical State of Gas}

We quantify the properties of dark matter halos, assuming a dynamically equilibrium state. The radius of dark matter halo, $r_{\mathrm{vir}}$, is estimated in terms of the mass of dark halo, $M_{\mathrm{vir}}$, and the redshift of virialization, $z_{\mathrm{vir}}$, as

$$
\frac{4}{3} \pi r_{\mathrm{vir}}^{3}\left\{1+\delta_{\mathrm{c}}\left(z_{\mathrm{vir}}\right)\right\} \rho_{\mathrm{c} 0} \Omega_{M}\left(1+z_{\mathrm{vir}}\right)^{3}=M_{\mathrm{vir}},
$$

where $\rho_{\mathrm{c} 0} \equiv 3 H_{0}^{2} / 8 \pi G$ is the critical density of the universe at $z=0, \delta_{c}\left(z_{\mathrm{vir}}\right)$ is the overdensity of a dark matter halo virialized at $z_{\text {vir }}$, and $G$ is the gravitational constant.

We assume dark matter halos as singular isothermal spheres and rotating uniform gas disks in their gravitational potentials. Cosmological $N$-body simulations show that structures of dark 
matter halos are described well by the Navarro-Frenk-White (NFW) profile (Navarro et al. 1996). Mo et al. (1998) studied a simple disk model in the gravitational potential of the singular isothermal sphere and a more realistic disk model in the gravitational potential of the NFW halo profile. We adopt a radius of the disk, $r_{\text {disk }} \simeq 0.18 r_{\text {vir }}$ (Ferrara et al. 2000; Hirashita $\&$ Ferrara 2002), by considering the conservation of angular momentum and assuming a typical value for the spin parameter $\lambda=0.04$ from the paper by Ferrara et al. (2000), who estimate the radius of the disk as $r_{\text {disk }}=4.5 \lambda r_{\text {vir }}$ in a modified isothermal halo.

In our one-zone model, we need a virial temperature for the initial gas temperature and a dynamical timescale of gas in the disk. Gas collapsed at $z_{\text {vir }}$ in the dark matter halo of $M_{\text {vir }}$ has a virial temperature, $T_{\text {vir }}$, defined as

$$
T_{\mathrm{vir}} \equiv \frac{G \mu \mathrm{m}_{\mathrm{H}} \mathrm{M}_{\mathrm{vir}}}{3 k_{\mathrm{B}} r_{\mathrm{vir}}},
$$

where $k_{\mathrm{B}}$ is the Boltzmann constant, $\mu$ is the mean molecular weight, and $m_{\mathrm{H}}$ is the mass of a hydrogen atom. The initial value for the temperature of gas, $T$, is assumed to be $T_{\text {vir }}$. A circular velocity, $v_{\mathrm{c}}$, is defined as

$$
v_{\mathrm{c}} \equiv\left(\frac{G M_{\mathrm{vir}}}{r_{\mathrm{vir}}}\right)^{\frac{1}{2}}
$$

and we also define a rotation timescale, $t_{\mathrm{cir}}$, as

$$
t_{\mathrm{cir}} \equiv \frac{2 \pi r_{\mathrm{disk}}}{v_{\mathrm{c}}}
$$

Note that the rotation timescale of the gas disk, $t_{\text {cir }}$, depends only on virialization redshift, $z_{\text {vir }}$, as

$$
t_{\mathrm{cir}}=9.3 \times 10^{7} \mathrm{yr}\left(\frac{11}{1+z_{\mathrm{vir}}}\right)^{\frac{3}{2}}\left(\frac{18 \pi^{2}}{\delta_{c}\left(z_{\mathrm{vir}}\right)}\right)^{\frac{1}{2}} .
$$

We must estimate the number density of the hydrogen gas, $n_{\mathrm{H}}$, because it affects both the chemical reaction rate and the cooling rate. The cooling time of halo gas is much shorter than the Hubble timescale for the objects of interest in this paper ( $T_{\text {vir }} \gtrsim 10000 \mathrm{~K}$; e.g., Hutchings et al. 2002). It is widely understood that most $z \sim 10$ galaxies are not clear disk galaxies; numerical simulations that proceed from cosmological initial conditions ( $z \sim 100-200$ ) clearly reveal that they possess highly irregular structures whose SFRs are not easily quantifiable, that filamentary accretion and frequent mergers are still churning the halo at this epoch, and that turbulent flows that arise in the center of the halo prevent coherent disks from forming on the spatial scales of galaxies (Johnson et al. 2008; Greif et al. 2010; Wise et al. 2010). We assume for simplicity that a significant fraction of baryons finally collapses to a disk in the dark matter halo potential. In semi-analytic models, it is assumed that the cooled halo gas settles into the disk (e.g., Cole et al. 2000). We make a similar assumption in our model, but a more detailed treatment for $\mathrm{H}_{2}$ formation, dust evolution, and star formation in the gas disk. The radius of disk, $r_{\text {disk }}$, is determined following Hirashita $\&$ Ferrara (2002). We estimate the typical scale height, $H$, from hydrostatic equilibrium (Shakura \& Sunyaev 1988)

$$
H=\sqrt{2} \frac{v_{\mathrm{s}}}{v_{\mathrm{c}}} r_{\mathrm{disk}}=\left(\frac{2 T}{3 T_{\mathrm{vir}}}\right)^{\frac{1}{2}} r_{\mathrm{disk}}
$$

for $H / r_{\text {disk }} \leqslant 0.1$; otherwise, we assume $H / r_{\text {disk }}=0.1$, where $v_{s}=\left(k_{B} T / \mu \mathrm{m}_{\mathrm{H}}\right)^{\frac{1}{2}}$ is the isothermal sound velocity. Therefore, the initial hydrogen density of disk, $n_{\mathrm{H}}$, is estimated as

$$
n_{\mathrm{H}}=\frac{M_{\mathrm{H}}}{\pi r_{\mathrm{disk}}^{2} 2 H m_{\mathrm{H}}} .
$$

The initial mass of hydrogen in the galaxy, $M_{\mathrm{H}}$, is written as

$$
\begin{aligned}
M_{\mathrm{H}} & =M_{\mathrm{gas}}-M_{\mathrm{He}}=M_{\mathrm{gas}} \frac{m_{\mathrm{H}}}{\left(m_{\mathrm{H}}+m_{\mathrm{He}} y_{\mathrm{He}}\right)} \\
& =\frac{M_{\mathrm{vir}} \Omega_{b}}{\Omega_{M}} \frac{m_{\mathrm{H}}}{\left(m_{\mathrm{H}}+m_{\mathrm{He}} y_{\mathrm{He}}\right)},
\end{aligned}
$$

where $M_{\mathrm{He}}$ is the initial mass of helium in the galaxy, $M_{\mathrm{gas}}=$ $M_{\mathrm{H}}+M_{\mathrm{He}}, m_{\mathrm{He}}$ is the mass of a helium atom, and $y_{\mathrm{He}}$ is the helium abundance. We assume $y_{\mathrm{He}}=0.0972$ (Omukai 2000). Note that since massive stars ionize surrounding gas and form an expanding $\mathrm{H}_{\mathrm{II}}$ region (e.g., Whalen et al. 2004; Kitayama et al. 2004), we adopt gas density around the SN progenitor, $n_{\mathrm{SN}}$, as being different from the hydrogen gas density in our one-zone galaxy model, $n_{\mathrm{H}}$. This is because the gas density around the $\mathrm{SN}$ progenitor, $n_{\mathrm{SN}}$, is closely related to the dust destruction process by reverse shocks driven by $\mathrm{SNe}$, as shown in Section 2.2.

\subsection{Star Formation Law}

We expect that the SFR, $\Psi(t)$, is roughly proportional to $t_{\mathrm{cir}}^{-1}$, since a representative timescale of the dynamics of the gas disk is $t_{\text {cir. }}$. We assume that

$$
\Psi(t)=\frac{f_{\mathrm{H}_{2}}(t) M_{\mathrm{H}}(t)}{t_{\mathrm{cir}}\left(z_{\mathrm{vir}}\right)},
$$

where $f_{\mathrm{H}_{2}}$ is the mass fraction of molecular hydrogen to the total gas. We should note that observationally Bigiel et al. (2008) find that $\mathrm{H}_{2}$ is converted into stars at a constant efficiency in nearby spirals, and Gnedin et al. (2009) show that the star formation recipe in a galaxy formation simulation in which star formation occurs only in the molecular gas can reproduce the observational correlations between SFR and the total gas density.

\subsection{Evolution of Gas, Stars, and Metals}

We calculate the time evolutions of the masses of hydrogen and helium gases, $M_{\text {gas }}$, stars, $M_{\text {star }}$, and metal of element $i$, $M_{\mathrm{m}, i}$, in the galaxy using the following equations:

$$
\begin{aligned}
\frac{d M_{\mathrm{gas}}(t)}{d t} & =-\Psi(t) \frac{M_{\mathrm{gas}}(t)}{M_{\mathrm{ISM}}(t)}+\overline{m_{\mathrm{gas}}} \gamma_{\mathrm{SN}}(t) \\
\frac{d M_{\mathrm{star}}(t)}{d t} & =\Psi(t)-\overline{m_{\mathrm{ejecta}}} \gamma_{\mathrm{SN}}(t) \\
\frac{d M_{\mathrm{m}, i}}{d t} & =-\Psi(t) \frac{M_{\mathrm{m}, i}(t)}{M_{\mathrm{ISM}}(t)}+\overline{m_{\mathrm{m}, i}} \gamma_{\mathrm{SN}}(t),
\end{aligned}
$$

where $M_{\mathrm{ISM}}(t)=M_{\mathrm{gas}}(t)+\sum_{i} M_{\mathrm{m}, i}(t), \overline{m_{\text {ejecta }}}=\overline{m_{\mathrm{gas}}}+$ $\sum_{i} \overline{m_{\mathrm{m}, i}}$ and $\overline{m_{\mathrm{gas}}}$ and $\overline{m_{\mathrm{m}, i}}$ are the gas mass of hydrogen and helium and the metal mass of element $i$ in SN ejecta, respectively. The mass returning to the ISM, $m_{\text {ejecta }}(m), m_{\text {gas }}(m)$, and $m_{\mathrm{m}, i}(m)$ through an SN with progenitor mass, $m$, is taken from Umeda \& Nomoto (2002) in the case of $m=13,20,25$ and $30 M_{\odot} \cdot \overline{m_{\text {gas }}}, \overline{m_{\text {ejecta }}}$, and $\overline{m_{m, i}}$ are IMF-averaged $m_{\text {gas }}(m)$, $m_{\text {ejecta }}(m)$, and $m_{\mathrm{m}, \mathrm{i}}(m)$, respectively. Note that metals consist not only of heavy elements in gas phase but also those in dust grains. 
Table 1

Reaction Rates Needed to Calculate the Abundance of $\mathrm{H}_{2}$

\begin{tabular}{|c|c|c|c|}
\hline No. & Reaction & $\begin{array}{c}\text { Rate } \\
\left(\mathrm{cm}^{3} \mathrm{~s}^{-1}\right)\end{array}$ & Ref. \\
\hline 1 & $\mathrm{H}+e^{-} \longrightarrow \mathrm{H}^{+}+2 e^{-}$ & $\begin{array}{l}\exp [-32.71+13.54 \ln (T(\mathrm{eV})) \\
-5.739(\ln (T(\mathrm{eV})))^{2}+1.563(\ln (T(\mathrm{eV})))^{3} \\
-0.2877(\ln (T(\mathrm{eV})))^{4}+3.483 \times 10^{-2}(\ln (T(\mathrm{eV})))^{5} \\
-2.632 \times 10^{-3}(\ln (T(\mathrm{eV})))^{6} \\
+1.120 \times 10^{-4}(\ln (T(\mathrm{eV})))^{7} \\
\left.-2.039 \times 10^{-6}(\ln (T(\mathrm{eV})))^{8}\right]\end{array}$ & 1 \\
\hline 2 & $\mathrm{H}^{+}+e^{-} \longrightarrow \mathrm{H}+\gamma$ & $\begin{array}{l}\exp [-28.61-0.7241(\ln (T(\mathrm{eV}))) \\
-2.026 \times 10^{-2}(\ln (T(\mathrm{eV})))^{2} \\
-2.381 \times 10^{-3}(\ln (T(\mathrm{eV})))^{3} \\
-3.213 \times 10^{-4}(\ln (T(\mathrm{eV})))^{4} \\
-1.422 \times 10^{-5}(\ln (T(\mathrm{eV})))^{5} \\
+4.989 \times 10^{-6}(\ln (T(\mathrm{eV})))^{6} \\
+5.756 \times 10^{-7}(\ln (T(\mathrm{eV})))^{7} \\
-1.857 \times 10^{-8}(\ln (T(\mathrm{eV})))^{8} \\
\left.-3.071 \times 10^{-9}(\ln (T(\mathrm{eV})))^{9}\right]\end{array}$ & 1 \\
\hline 3 & $\mathrm{H}+e^{-} \longrightarrow \mathrm{H}^{-}+\gamma$ & $1.4 \times 10^{-18} T^{0.928} \exp \left(-T / 1.62 \times 10^{4}\right)$ & 1 \\
\hline 4 & $\mathrm{H}^{-}+\mathrm{H} \longrightarrow \mathrm{H}_{2}+e^{-}$ & $\begin{array}{l}4.0 \times 10^{-9} T^{-0.17}(T>300) \\
1.5 \times 10^{-9}(T<300)\end{array}$ & 1 \\
\hline 5 & $\mathrm{H}^{-}+\mathrm{H}^{+} \longrightarrow 2 \mathrm{H}$ & $\begin{array}{l}5.7 \times 10^{-6} T^{-1 / 2}+6.3 \times 10^{-8} \\
-9.2 \times 10^{-11} T^{1 / 2}+4.4 \times 10^{-13} T\end{array}$ & 1 \\
\hline 6 & $\mathrm{H}+\mathrm{H}^{+} \longrightarrow \mathrm{H}_{2}^{+}+\gamma$ & $\begin{array}{l}\operatorname{dex}\left[-19.38-1.523 \log _{10} T\right. \\
\left.+1.118\left(\log _{10} T\right)^{2}-0.1269\left(\log _{10} T\right)^{3}\right]\end{array}$ & 1 \\
\hline 7 & $\mathrm{H}_{2}^{+}+\mathrm{H} \longrightarrow \mathrm{H}_{2}+\mathrm{H}^{+}$ & $6.4 \times 10^{-10}$ & 1 \\
\hline 8 & $\mathrm{H}_{2}^{+}+e^{-} \longrightarrow 2 \mathrm{H}$ & $2.0 \times 10^{-7} T^{-1 / 2}$ & 1 \\
\hline 9 & $\mathrm{H}_{2}+\mathrm{H}^{+} \longrightarrow \mathrm{H}_{2}^{+}+\mathrm{H}$ & $\begin{array}{l}3.0 \times 10^{-10} \exp (-21050 / T)\left(T<10^{4}\right) \\
1.5 \times 10^{-10} \exp (-14000 / T)\left(T>10^{4}\right)\end{array}$ & 2 \\
\hline 10 & $\mathrm{H}_{2}+\mathrm{H} \longrightarrow 3 \mathrm{H}$ & $\begin{array}{l}k_{\mathrm{H}}^{1-a} k_{\mathrm{L}}^{a} \\
k_{\mathrm{L}}=1.12 \times 10^{-10} \exp \left(-7.035 \times 10^{4} / T\right) \\
k_{\mathrm{H}}=6.5 \times 10^{-7} T^{-1 / 2} \\
\times \exp \left(-5.2 \times 10^{4} / T\right)[1-\exp (-6000 / T)] \\
a=4.0-0.416 \log _{10}\left(T / 10^{4}\right)-0.327\left(\log _{10}\left(T / 10^{4}\right)\right)^{2}\end{array}$ & 1 \\
\hline 11 & $\mathrm{H}_{2}+e^{-} \longrightarrow 2 \mathrm{H}+e^{-}$ & $4.4 \times 10^{-10} T^{0.35} \exp \left(-1.02 \times 10^{5} / T\right)$ & 1 \\
\hline Dust & $\mathrm{H}+\mathrm{H}+$ grain $\longrightarrow \mathrm{H}_{2}+$ grain & See Section 3.5 & \\
\hline
\end{tabular}

Note. The unit of the gas temperature $T$ is K unless otherwise stated.

References. (1) Omukai 2000; (2) Galli \& Palla 1998.

\subsection{Chemistry and Cooling}

We follow the time evolution of molecular mass fraction, $f_{\mathrm{H}_{2}}$, ionization degree, $x$, and gas temperature, $T$. We define the molecular fraction of hydrogen as

$$
f_{\mathrm{H}_{2}} \equiv \frac{2 n_{\mathrm{H}_{2}}}{n_{\mathrm{H}}},
$$

where $n_{\mathrm{H}_{2}}$ and $n_{\mathrm{H}}$ are the number densities of molecular hydrogen and hydrogen nuclei, respectively. The molecular fraction is very important in our models, because it determines the final cooling rate of low-metallicity gas. The metal-free gas evolution with chemical reactions and cooling is studied using the model by Tegmark et al. (1997), Hutchings et al. (2002), and Hirashita \& Ferrara (2002). In Table 1, we summarize chemical reactions considered in this paper and their rate coefficients $\left(R_{n} ; n=1, \ldots, 11\right)$. The equations are based on Hirashita $\&$ Ferrara (2002), but we include the effect of the dust size distribution on $\mathrm{H}_{2}$ formation and the metal-line cooling process.

The time evolution of the ionization degree is described as

$$
\frac{d x}{d t}=x f_{0} R_{1} n_{\mathrm{H}}-x^{2} R_{2} n_{\mathrm{H}}+\Gamma_{12} f_{0},
$$

where $f_{0}=1-x-f_{\mathrm{H}_{2}}$ is the neutral fraction of hydrogen. The terms on the right-hand side are the rates of collisional ionization, recombination, and photoionization. Next, the time evolution of the molecular fraction is written as

$$
\begin{aligned}
\frac{d f_{\mathrm{H}_{2}}}{d t}= & {\left[\frac{d f_{\mathrm{H}_{2}}}{d t}\right]_{\mathrm{gas}}+\left[\frac{d f_{\mathrm{H}_{2}}}{d t}\right]_{\mathrm{dust}}+\left[\frac{d f_{\mathrm{H}_{2}}}{d t}\right]_{\mathrm{dest}} } \\
& +\left[\frac{d f_{\mathrm{H}_{2}}}{d t}\right]_{\mathrm{UV}}+\left[\frac{d f_{\mathrm{H}_{2}}}{d t}\right]_{\mathrm{star}},
\end{aligned}
$$

where the terms on the right-hand side are the $\mathrm{H}_{2}$ formation rate in the gas phase, the $\mathrm{H}_{2}$ formation rate on dust grains, the destruction rate in the gas phase, the destruction rate by UV photons, and the decreasing rate by star formation, respectively. These terms are given by

$$
\begin{aligned}
& {\left[\frac{d f_{\mathrm{H}_{2}}}{d t}\right]_{\mathrm{gas}}=2 f_{0}^{2} x n_{\mathrm{H}}\left(R_{\mathrm{eff}, 1}+R_{\mathrm{eff}, 2}\right),} \\
& {\left[\frac{d f_{\mathrm{H}_{2}}}{d t}\right]_{\mathrm{dust}}=2 R_{\mathrm{dust}} \mathcal{D} n_{\mathrm{H}} f_{0},} \\
& {\left[\frac{d f_{\mathrm{H}_{2}}}{d t}\right]_{\mathrm{dest}}=-f_{\mathrm{H}_{2}} n_{\mathrm{H}}\left(x^{2} R_{\mathrm{eff}, 3}+f_{0} R_{10}+x R_{11}\right),} \\
& {\left[\frac{d f_{\mathrm{H}_{2}}}{d t}\right]_{\mathrm{UV}}=-\Gamma_{13} f_{\mathrm{H}_{2}},}
\end{aligned}
$$


and

$$
\left[\frac{d f_{\mathrm{H}_{2}}}{d t}\right]_{\mathrm{star}}=-\left(1-f_{\mathrm{H}_{2}}\right) \Psi(t) \frac{1}{M_{\mathrm{ISM}}(t)} .
$$

The effective formation rates of $\mathrm{H}_{2}$ including the effect of the destruction rate of $\mathrm{H}^{-}$and $\mathrm{H}_{2}^{+}$are

$$
R_{\mathrm{eff}, 1} \equiv \frac{R_{3} R_{4}}{f_{0} R_{4}+x R_{5}+\Gamma_{14} / n_{\mathrm{H}}}
$$

and

$$
R_{\mathrm{eff}, 2} \equiv \frac{R_{6} R_{7}}{f_{0} R_{7}+x R_{8}+\Gamma_{15} / n_{\mathrm{H}}},
$$

respectively, and the destruction of $\mathrm{H}_{2}^{+}$due to $\mathrm{H}^{-}$collision is

$$
R_{\mathrm{eff}, 3} \equiv \frac{R_{8} R_{9}}{f_{0} R_{7}+x R_{8}+\Gamma_{15} / n_{\mathrm{H}}} .
$$

We will give the dust-to-gas mass ratio, $\mathcal{D}$, and the production rate of molecular hydrogen via dust surface reaction, $R_{\text {dust }}$, in Section 3.5 and reaction rates of the photo-process, $\Gamma_{n}(n=$ $12, \ldots, 15)$, in Section 3.6.

At temperature $<10^{4} \mathrm{~K}$, the main coolant is molecular hydrogen in low-metallicity gas. The cooling rate for molecular hydrogen, $\Lambda_{\mathrm{H}_{2}}$, over the range $10 \mathrm{~K} \leqslant T \leqslant 10^{4} \mathrm{~K}$ is given by (Galli \& Palla 1998)

$$
\begin{aligned}
\log _{10}\left(\frac{\Lambda_{\mathrm{H}_{2}}(T)}{n_{\mathrm{H}} n_{\mathrm{H}_{2}} \mathrm{erg} \mathrm{cm}^{3} \mathrm{~s}^{-1}}\right)= & -103.0+97.59 T_{\log }-48.05 T_{\log }^{2} \\
& +10.80 T_{\log }^{3}-0.9032 T_{\log }^{4}, \quad(27)
\end{aligned}
$$

where $T_{\log } \equiv \log _{10}(T / \mathrm{K})$. Glover \& Abel (2008) have recently given the $\mathrm{H}_{2}$ cooling rates, which include $\mathrm{H}-\mathrm{H}_{2}$ collision and $\mathrm{H}_{2}-\mathrm{H}_{2}$ collision pathways, while the Galli \& Palla (1998) rates include only $\mathrm{H}-\mathrm{H}_{2}$ collisions. We assume that the lower limit of gas temperature is the CMB temperature.

At temperature $T \gtrsim 10^{4} \mathrm{~K}$, collisional excitation, $\Lambda_{\mathrm{H}, \text { ce }}$, and (less importantly) ionization of atomic hydrogen, $\Lambda_{\mathrm{H}, \mathrm{ci}}$, are more dominant cooling processes than molecular hydrogen cooling and are given by (Haiman et al. 1996)

$$
\frac{\Lambda_{\mathrm{H}, \mathrm{ce}}(T)}{n_{\mathrm{e}^{-} n_{\mathrm{H}}} \operatorname{erg~cm}^{3} \mathrm{~s}^{-1}}=7.50 \times 10^{-19} \frac{1}{1+T_{5}^{\frac{1}{2}}} \exp ^{-\frac{1.183}{T_{5}}}
$$

and

$$
\frac{\Lambda_{\mathrm{H}, \mathrm{ci}}(T)}{n_{\mathrm{e}^{-} n_{\mathrm{H}}} \operatorname{erg~cm}^{3} \mathrm{~s}^{-1}}=4.02 \times 10^{-19} \frac{T_{5}^{\frac{1}{2}}}{1+T_{5}^{\frac{1}{2}}} \exp ^{-\frac{1.578}{T_{5}}},
$$

respectively, where $T_{5}$ is the gas temperature in units of $10^{5} \mathrm{~K}$.

We consider fine-structure cooling by $\mathrm{C}_{\mathrm{I}}, \mathrm{C}_{\mathrm{II}}$, and $\mathrm{O}_{\mathrm{I}}$, which dominates the thermal evolution for the number density of gas of interest in this paper (Omukai et al. 2005). The related parameters of transitions are given in Hollenbach \& McKee (1989).

\subsection{Formation of Molecular Hydrogen on Dust Grains}

The increasing rate of molecular fraction via dust surface reaction is estimated as

$$
\left[\frac{d f_{\mathrm{H}_{2}}}{d t}\right]_{\mathrm{dust}}=2 R_{\mathrm{dust}} \mathcal{D} n_{\mathrm{H}} f_{0}=\sum_{j} \int_{0}^{\infty} f_{0} f_{j}(a) \pi a^{2} \bar{v} S d a,
$$

where $\bar{v}$ is the mean thermal speed of hydrogen and $S$ is the sticking efficiency of hydrogen atoms. We assume that the gas follows a Maxwellian distribution so that thermal speed is given by (Krügel 2008)

$$
\bar{v}=\left(\frac{8}{\pi} \frac{k_{\mathrm{B}} T}{m_{\mathrm{H}}}\right)^{\frac{1}{2}}
$$

Here, we define the dust-to-gas mass ratio, $\mathcal{D}$, as

$$
\mathcal{D} \equiv \sum_{j} \int_{0}^{\infty} \frac{4 \pi a^{3} \rho_{j} f_{j}(a)}{3 n_{\mathrm{H}} m_{\mathrm{H}}} d a .
$$

The reaction rate of the $\mathrm{H}_{2}$ formation on grains, $R_{\text {dust }}$, can be estimated as

$$
R_{\text {dust }}(a) \mathcal{D}=\sum_{j} \int_{0}^{\infty}\left(\frac{3 m_{\mathrm{H}} \bar{v} S}{8 a \rho_{j}}\right)\left(\frac{4 \pi a^{3} \rho_{j} f_{j}(a)}{3 n_{\mathrm{H}} m_{\mathrm{H}}}\right) d a .
$$

We adopt $S=0.2$ for $T<300 \mathrm{~K}$ and $S=0$ for $T>300 \mathrm{~K}$ (Hirashita \& Ferrara 2002).

\subsection{Radiative Properties}

We follow photo-processes in chemical reactions and heating processes in thermal evolution by using the models developed by Kitayama \& Ikeuchi (2000) and improved in Hirashita \& Ferrara (2002). The intrinsic luminosity is assumed to be equal to the total luminosity of OB stars whose mass is larger than $3 M_{\odot}(\operatorname{Cox} 2000)$ :

$$
L_{\mathrm{UV}, 0}(t)=\int_{3 M_{\odot}}^{\infty} d m \int_{0}^{\tau_{m}} d t^{\prime} L(m) \phi(m) \Psi\left(t-t^{\prime}\right),
$$

where $L(m)$ is the stellar luminosity as a function of stellar mass $m$. For $L(m)$, we adopt the model of zero-metallicity stars without mass loss in Schaerer (2002). We assume the spectrum of the incident UV radiation from stars is a power law with an index $\alpha$ :

$$
I_{\mathrm{UV}}(v)=I_{0}\left(v_{\mathrm{HI}}\right)\left(\frac{v}{v_{\mathrm{HI}}}\right)^{-\alpha},
$$

where $v$ is the frequency of photons and $I_{0}\left(v_{\mathrm{HI}}\right)$ is the intensity at the ionization threshold frequency of neutral hydrogen $v_{\mathrm{H}_{\mathrm{I}}}=$ $3.3 \times 10^{15} \mathrm{~Hz}$. In this paper, we simply set $\alpha=5$ according to Hirashita \& Ferrara (2002). The normalization of the intensity is determined by

$$
\frac{L_{\mathrm{UV}, 0} \exp \left(-\tau_{\mathrm{disk}}\right)}{4 \pi r_{\text {disk }}^{2}}=\int_{v_{\min }}^{\infty} I_{\mathrm{UV}}(\nu) d \nu
$$

where $v_{\min }$ is the minimum frequency where OB stars dominate the radiative energy of star-forming galaxies, and $\tau_{\text {disk }}$ is the typical dust optical depth in the disk. We assume that $v_{\min }=$ $10^{15} \mathrm{~Hz}$. This typical optical depth can be simply estimated as

$$
\tau_{\mathrm{disk}}=r_{\mathrm{disk}} \sum_{j} \int_{0}^{\infty} \pi a^{2} f_{j}(a) d a
$$

by assuming that extinction efficiency of dust is unity in UV. We calculate $\Gamma_{12}, \Gamma_{14}$, and $\Gamma_{15}$ from Equation (A20) of Kitayama \& Ikeuchi (2000) and the heating rate from Equation (A21) of Kitayama \& Ikeuchi (2000). We summarize the cross section for the photo-process in Table 2 . The $\mathrm{H}_{2}$ photodissociation cross 
Table 2

Cross Sections for the Photoionization and Photodissociation Process, where the Frequency, $v$, is in Units of $\mathrm{Hz}$

\begin{tabular}{|c|c|c|c|c|}
\hline No. & Reaction & $\begin{array}{l}\text { Cross Section } \\
\quad\left(\mathrm{cm}^{2}\right)\end{array}$ & $\begin{array}{l}v \text { Range } \\
(\mathrm{Hz})\end{array}$ & Ref. \\
\hline 12 & $\mathrm{H}+\gamma \longrightarrow \mathrm{H}^{+}+e^{-}$ & $6.30 \times 10^{-18}\left(v / 3.3 \times 10^{15}\right)^{-3.0}$ & $v>3.3 \times 10^{15}$ & 1 \\
\hline 13 & $\mathrm{H}_{2}+\gamma \longrightarrow \mathrm{H}_{2}^{*} \longrightarrow 2 \mathrm{H}$ & see Equation (38) & & 2 \\
\hline 14 & $\mathrm{H}^{-}+\gamma \longrightarrow \mathrm{H}+e^{-}$ & $\begin{array}{l}3.486 \times 10^{-16}(x-1)^{3 / 2} / x^{3.11} \\
\left(x \equiv v / 1.8 \times 10^{14}\right)\end{array}$ & $v>1.8 \times 10^{14}$ & 3 \\
\hline 15 & $\mathrm{H}_{2}^{+}+\gamma \longrightarrow \mathrm{H}+\mathrm{H}^{+}$ & $\begin{array}{l}7.401 \times 10^{-18} \\
\operatorname{dex}\left(-x^{2}-0.0302 x^{3}-0.0158 x^{4}\right) \\
\left(x \equiv 2.762 \ln \left(v / 2.7 \times 10^{15}\right)\right.\end{array}$ & $v>6.4 \times 10^{14}$ & 3 \\
\hline
\end{tabular}

References. (1) Kitayama \& Ikeuchi 2000; (2) Abel et al. 1997; (3) Tegmark et al. 1997.

section is given by Abel et al. (1997). However, if the $\mathrm{H}_{2}$ column density becomes larger than $10^{14} \mathrm{~cm}^{-2}$, self-shielding effects become important (Draine \& Bertoldi 1996). Therefore, the $\mathrm{H}_{2}$ dissociation rate, $\Gamma_{13}$, is given by (Hirashita \& Ferrara 2002)

$$
\begin{aligned}
\Gamma_{13}= & (4 \pi) 1.1 \times 10^{8} I_{\mathrm{UV}}\left(3.1 \times 10^{15} \mathrm{~Hz}\right) \\
& \times\left(\frac{n_{\mathrm{H}} f_{\mathrm{H}_{2}} r_{\text {disk }}}{10^{14} \mathrm{~cm}^{-2}}\right)^{-0.75} \mathrm{~s}^{-1},
\end{aligned}
$$

where $I_{\mathrm{UV}}\left(3.1 \times 10^{15} \mathrm{~Hz}\right)$ is in the Lyman-Werner band. We should note that adoption of $r_{\text {disk }}$ in Equation (38) gives an extreme upper bound to the self-shielding, so we may overestimate self-shielding to internal Lyman-Werner photons by $\mathrm{H}_{2}$. In this paper, we focus on the effects of dust on the protogalaxy, so for simplicity, we set an extreme upper bound to the self-shielding.

We do not consider the Lyman-Werner background, since we concentrate on the evolution of atomic line cooling halos $\left(M_{\mathrm{vir}}>10^{8} M_{\odot}\right.$ in $\left.z<10\right)$ in which destruction of molecular hydrogen by the Lyman-Werner background is less efficient (O'Shea \& Norman 2008; Susa 2008; Wise \& Abel 2008; Wise $\&$ Cen 2009). However, $10^{8}-10^{9} M_{\odot}$ halos are not immune to the Lyman-Werner background, just self-shielded at their very centers. Not all the baryons will be protected from external photodissociating flux, and this will affect $\mathrm{H}_{2}$ production on dust outside the center of halo. In the lower mass halos, the Lyman-Werner background may be effective to dissociate $\mathrm{H}_{2}$ molecules (Machacek et al. 2001, 2003; Yoshida et al. 2003; Susa 2007; Wise \& Abel 2007; O’Shea \& Norman 2008).

\section{RESULTS}

Our fiducial model assumes $M_{\mathrm{vir}}=10^{9} M_{\odot}$ and $z_{\mathrm{vir}}=10$ and includes the dust destruction model by both reverse shocks and forward shocks with the ISM density around the SN progenitor, $n_{\mathrm{SN}}=1 \mathrm{~cm}^{-3}$ (see Table 3 for a summary of our models). This dark matter halo forms from a $2.5 \sigma$ density fluctuation. We stop the calculation at $z=5$. It corresponds to the galaxy age of $\sim 0.8 \mathrm{Gyr}$; that is, before SN II becomes a dominant source of dust grains. The initial mass of gas is $M_{\text {gas }}=1.7 \times 10^{8} M_{\odot}$ and the dynamical timescale of circular motion of the gas disk is $t_{\text {cir }}\left(z_{\text {vir }}=10\right)=9.5 \times 10^{7} \mathrm{yr}$. Note that galaxies with $M_{\mathrm{vir}} \sim 10^{9} M_{\odot}$ play a critical role in the cosmic reionization, since in the relevant redshift range for cosmological reionization, $z=6-15$; most of the reionization radiation is expected to come from galaxies with masses less than $\sim 10^{9.5} M_{\odot}$ (Wise \& Cen 2009).
Table 3

The Dust Destruction Model and the Main Parameters

\begin{tabular}{llcc}
\hline \hline Model & Dust Destruction & $\begin{array}{c}n_{\mathrm{SN}} \\
\left(\mathrm{cm}^{-3}\right)\end{array}$ & $\begin{array}{c}M_{\mathrm{vir}} \\
\left(M_{\odot}\right)\end{array}$ \\
\hline C1m9 & No destruction & $\ldots$ & $10^{9}$ \\
B1m9 & Forward shocks & 1 & $10^{9}$ \\
A1m9 (fiducial) & Forward and reverse shocks & 1 & $10^{9}$ \\
A0.03m9 & Forward and reverse shocks & 0.03 & $10^{9}$ \\
A0.1m9 & Forward and reverse shocks & 0.1 & $10^{9}$ \\
A0.3m9 & Forward and reverse shocks & 0.3 & $10^{9}$ \\
A3m9 & Forward and reverse shocks & 3 & $10^{9}$ \\
A10m9 & Forward and reverse shocks & 10 & $10^{9}$ \\
A0.1m8 & Forward and reverse shocks & 0.1 & $10^{8}$ \\
A0.1m10 & Forward and reverse shocks & 0.1 & $10^{10}$ \\
A0.1m11 & Forward and reverse shocks & 0.1 & $10^{11}$ \\
A1m8 & Forward and reverse shocks & 1 & $10^{8}$ \\
A1m10 & Forward and reverse shocks & 1 & $10^{10}$ \\
A1m11 & Forward and reverse shocks & 1 & $10^{11}$ \\
A10m8 & Forward and reverse shocks & 10 & $10^{8}$ \\
A10m10 & Forward and reverse shocks & 10 & $10^{10}$ \\
A10m11 & Forward and reverse shocks & 10 & $10^{11}$ \\
\hline
\end{tabular}

\subsection{The Dust Destruction}

We first show the evolution of a galaxy with $M_{\text {vir }}=10^{9} M_{\odot}$ and $z_{\text {vir }}=10$ for various dust destruction models. To clarify the dust destruction effects on galaxy evolution, we first show the result of the model without reverse and forward shocks (model C1m9), then compare the results of the models with only forward shocks (model B1m9) and with both forward and reverse shocks (model A1m9) to the model without both shocks (model C1m9).

In Figure 5, we show the evolution of various quantities without dust destruction (model $\mathrm{C} 1 \mathrm{~m} 9$ ). The figure shows the time evolution of the molecular fraction, $f_{\mathrm{H}_{2}}$; the SFR in units of $M_{\odot} \mathrm{yr}^{-1}, \Psi$; the stellar mass fraction, $M_{\text {star }} /\left(M_{\text {gas }}+M_{\text {star }}\right)$; the metallicity, $Z$, in units of $Z_{\odot}$; total dust cross section per unit volume, $\sigma_{d,-20}$, in units of $10^{-20} \mathrm{~cm}^{-1}$; and the dust-to-gas mass ratio, $D_{d,-2}$, in units of $10^{-2}$. The definition of $D_{d,-2}$ is convenient for comparison with the MW value of the dust-to-gas mass ratio. In the $\mathrm{MW}$, the dust-to-gas mass ratio is $0.5 \times 10^{-2}$ in the diffuse ISM (Draine 2009) and $0.9 \times 10^{-2}$ in molecular clouds (Pollack et al. 1994).

The molecular fraction, $f_{\mathrm{H}_{2}}$, is very important, since it determines SFR and controls galaxy evolution. The molecular fraction reaches $f_{\mathrm{H}_{2}} \sim 1 \times 10^{-3}$ around $t \sim 10^{7} \mathrm{yr}$. This result is robust for all models, since in this stage $\mathrm{H}_{2}$ formation in the gas phase is dominant over that on dust grains (Tegmark et al. 1997; Hirashita \& Ferrara 2002). The gas temperature 


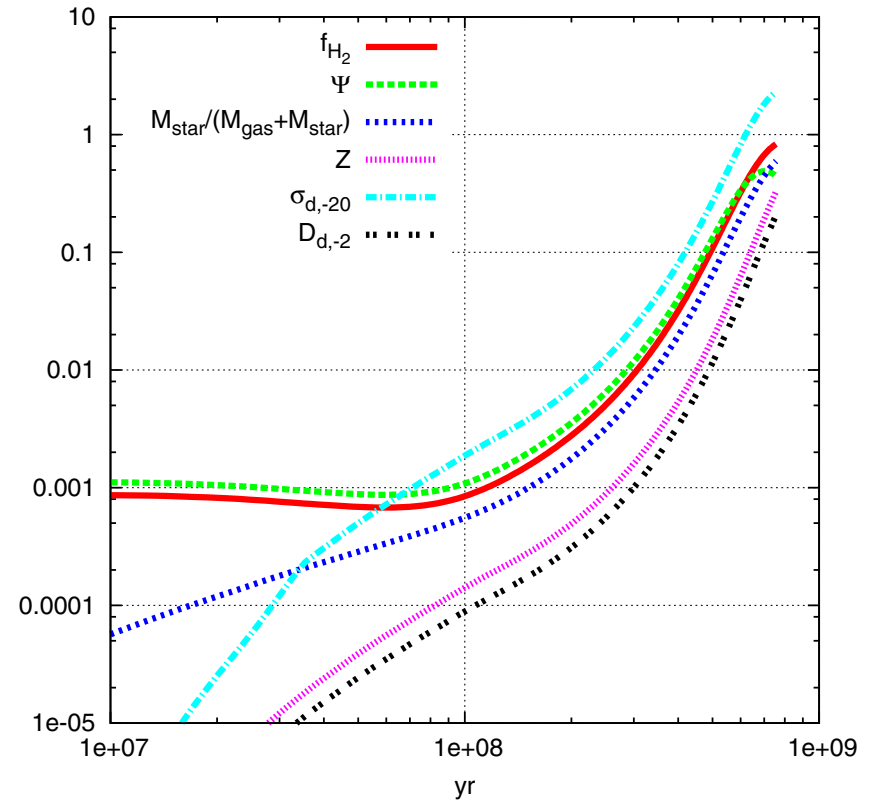

Figure 5. Time evolution for model $\mathrm{C} 1 \mathrm{~m} 9$, in which dust destruction by the reverse and forward shocks is not considered. We show molecular fraction, $f_{\mathrm{H}_{2}}$; SFR in units of $M_{\odot} \mathrm{yr}^{-1}, \Psi$; star-to-gas mass ratio, $M_{\mathrm{star}} /\left(M_{\mathrm{gas}}+M_{\mathrm{star}}\right)$; metallicity, $Z$, in units of $Z_{\odot}$; total dust cross section per unit volume, $\sigma_{d,-20}$, in units of $10^{-20} \mathrm{~cm}^{-1}$; and dust-to-gas mass ratio, $D_{d,-2}$, in units of $10^{-2}$. The horizontal axis shows the age of the galaxy in units of yr from the formation redshift, $z_{\text {vir }}=10$.

(A color version of this figure is available in the online journal.)

rapidly drops below $200 \mathrm{~K}$ before $10^{7} \mathrm{yr}$. Then, the molecular fraction rapidly increases from $t \sim 10^{8} \mathrm{yr}$ and reaches $\sim 0.83$ at the galaxy age of $\sim 0.8 \mathrm{Gyr}(z=5)$. This is due to the enhancement of $\mathrm{H}_{2}$ formation on dust grains by an increase of $\sigma_{d,-20}$. For $t \gtrsim 3 \times 10^{7} \mathrm{yr}, \sigma_{d,-20} \gtrsim 1.1 \times 10^{-4}$ and $\left[d f_{\mathrm{H}_{2}} / d t\right]_{\text {dust }}$ exceeds $\left[d f_{\mathrm{H}_{2}} / d t\right]_{\text {star }}$. For $\sigma_{d,-20} \gtrsim 0.001$, the increase of the molecular fraction enhances the star formation. The cycle of the $\mathrm{H}_{2}$ formation on dust, the star formation, and the dust formation by SNe significantly accelerates galaxy evolution by, for example, rapidly increasing the stellar mass fraction, $M_{\text {star }} /\left(M_{\text {gas }}+M_{\text {star }}\right)$. At the galaxy age $\sim 0.8 \times 10^{9} \mathrm{yr}$, the stellar mass fraction goes up to $M_{\text {star }} /\left(M_{\text {gas }}+M_{\text {star }}\right) \sim 0.60$. The SFR, $\Psi(t)$, decreases from the time when $M_{\text {star }} /\left(M_{\text {gas }}+M_{\text {star }}\right) \sim 0.45$, since gas mass decreases significantly. The active star formation causes the formation of dust grains and metals. At $t \sim 0.8 \mathrm{Gyr}$, the total dust cross section, $\sigma_{d,-20}$, goes up to 2.3 and the metallicity, $Z$, goes up to $3.3 \times 10^{-1} Z_{\odot}$.

Figure 6 shows the results of the galaxy model with the dust destruction by only the forward shocks (model B1m9) to illustrate the effects of dust destruction by forward shocks on the galaxy evolution. In this model $n_{\mathrm{SN}}=1.0 \mathrm{~cm}^{-3}$. The dust destruction by forward shocks slightly affects the dust-to-gas mass ratio, $D_{d,-2}$, after the galaxy age of $\sim 5 \times$ $10^{8} \mathrm{yr}$. This is because the destruction by forward shocks is roughly proportional to the dust-to-gas mass ratio (see Equation (9)), and dust grains are destroyed significantly for $D_{d,-2} \gtrsim 0.1$ in this case. The SFR decreases from the time when $M_{\text {star }} /\left(M_{\text {gas }}+M_{\text {star }}\right) \sim 0.4$. At $\sim 0.8 \mathrm{Gyr}$, the molecular fraction reaches $f_{\mathrm{H}_{2}} \sim 0.51$ and the stellar mass fraction reaches $M_{\text {star }} /\left(M_{\text {gas }}+M_{\text {star }}\right) \sim 0.47$.

In Figure 7 , we show the results of our fiducial model A $1 \mathrm{~m} 9$ in which we include the dust destruction by both reverse and forward shocks in the case of $n_{\mathrm{SN}}=1 \mathrm{~cm}^{-3}$. The

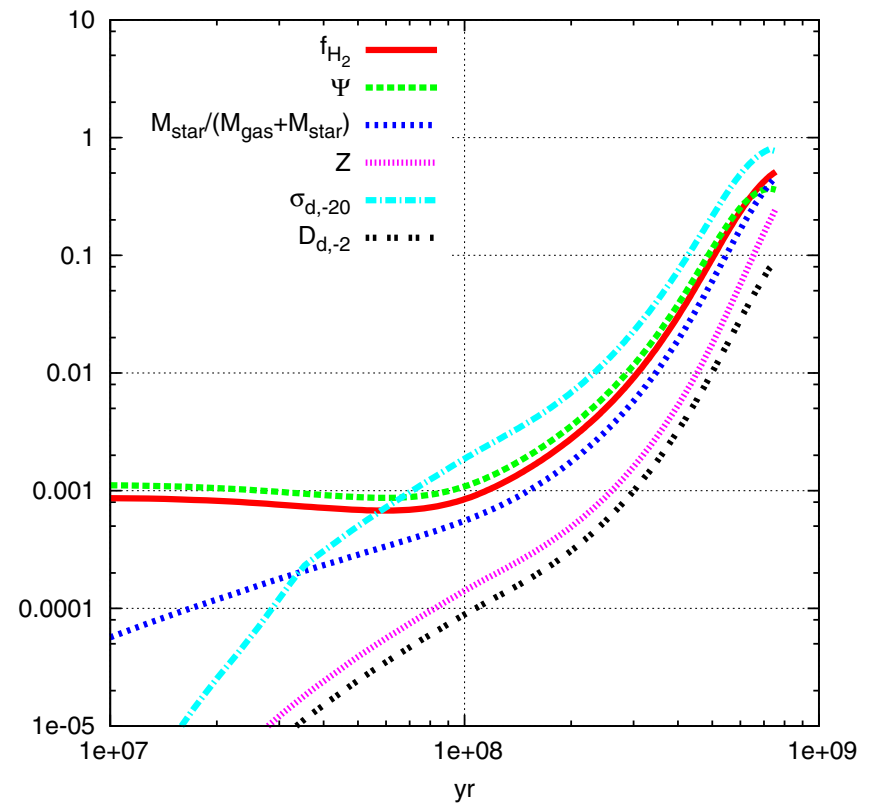

Figure 6. Same as Figure 5 but for model B $1 \mathrm{~m} 9$, in which only the dust destruction by the forward shocks is considered.

(A color version of this figure is available in the online journal.)

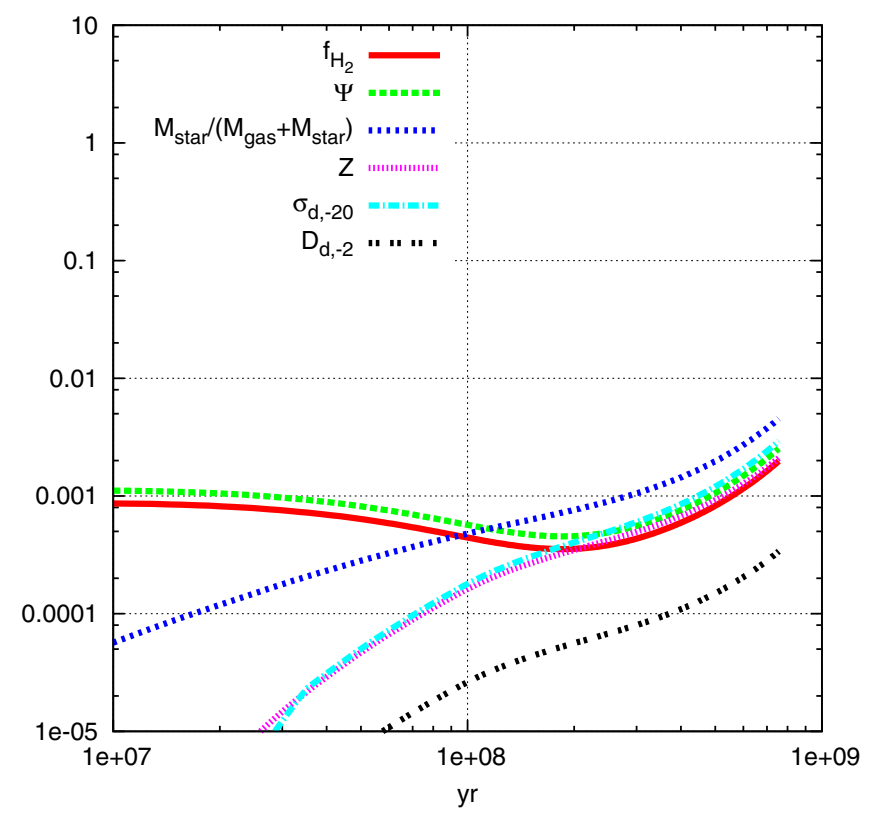

Figure 7. Same as Figure 5 but for model A1m9, in which the destruction by reverse and forward shocks in the case of $n_{\mathrm{SN}}=1 \mathrm{~cm}^{-3}$ is considered.

(A color version of this figure is available in the online journal.)

molecular fraction reaches $f_{\mathrm{H}_{2}} \sim 1 \times 10^{-3}$ around $t \sim 10^{7} \mathrm{yr}$. This is similar to $\mathrm{C} 1 \mathrm{~m} 9$ and $\mathrm{B} 1 \mathrm{~m} 9$. After $t \gtrsim 10^{7} \mathrm{yr}$, the molecular fraction evolution is quite different from models $\mathrm{C} 1 \mathrm{~m} 9$ and $\mathrm{B} 1 \mathrm{~m} 9$. The molecular fraction declines slowly until $t \sim 2 \times 10^{8} \mathrm{yr}$. After $\sim 2 \times 10^{8} \mathrm{yr}$, the molecular fraction increases slowly with increase of the dust mass. This is due to the $\mathrm{H}_{2}$ formation on the dust grains. The molecular fraction reaches only $\sim 2.0 \times 10^{-3}$ at $t \sim 0.8 \mathrm{Gyr}$. This is because dust destruction by reverse shocks is very effective and hence results in suppression of $\mathrm{H}_{2}$ formation on dust grains. On the other hand, forward shocks hardly affect the evolution of dust size and dust mass, since the destruction of forward shocks 


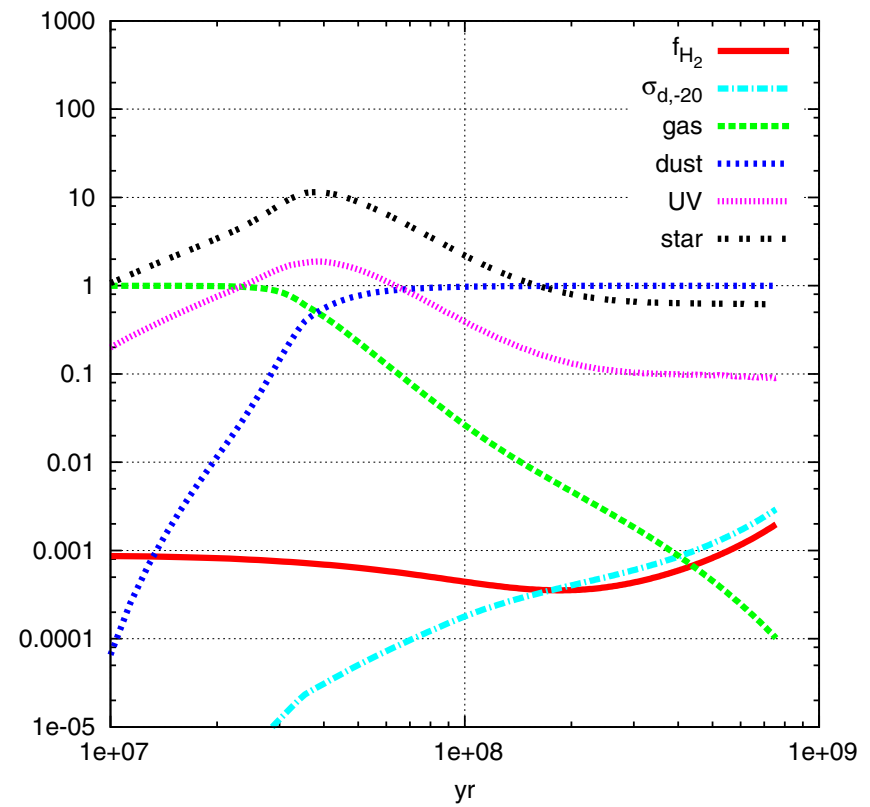

Figure 8. Formation and destruction rates of $\mathrm{H}_{2}$ in model $\mathrm{A} 1 \mathrm{~m} 9$. The evolution of the molecular formation rate in the gas phase (gas); the molecular formation rate on dust grains (dust); the molecular destruction rate by UV photons (UV); and the molecular decreasing rate by star formation (star), normalized to the total formation rate, are shown. The molecular formation on dust grains becomes dominant, compared with the other channels, after $t \sim 1.6 \times 10^{8} \mathrm{yr}$. The molecular fraction, $f_{\mathrm{H}_{2}}$, and total dust cross section per unit volume in units of $10^{-20} \mathrm{~cm}^{-1}, \sigma_{d,-20}$, are the same as in Figure 7 .

(A color version of this figure is available in the online journal.)

can change dust mass only for a large dust-to-gas mass ratio, $D_{d,-2} \gtrsim 10^{-1}$. At $t \sim 0.8 \mathrm{Gyr}$, the stellar mass fraction reaches only $M_{\text {star }} /\left(M_{\text {gas }}+M_{\text {star }}\right) \sim 4.5 \times 10^{-3}$, which is much less than the model without reverse shocks shown in Figure 5 (model C1m9) and 6 (model B1m9).

We illustrate the difference in the $\mathrm{H}_{2}$ formation rate among models $\mathrm{C} 1 \mathrm{~m} 9, \mathrm{~B} 1 \mathrm{~m} 9$, and $\mathrm{A} 1 \mathrm{~m} 9$ as follows: The $\mathrm{H}_{2}$ formation rate depends not only on the total dust mass but also on the dust size distribution. In models $\mathrm{C} 1 \mathrm{~m} 9$ and $\mathrm{B} 1 \mathrm{~m} 9$, the dust mass produced by an SN II without reverse shock is $\sum_{j} \int \overline{\mathcal{M}_{d, j}^{0}}(a) d a=0.48 M_{\odot}$ and in A1m9, the dust mass injection into ISM through a reverse shock with $n_{\mathrm{SN}}=1.0 \mathrm{~cm}^{-3}$ is $\sum_{j} \int \overline{\mathcal{M}_{d, j}^{1.0}}(a) d a=0.15 M_{\odot}$. The ratio of the mean dust area to the mean dust volume is $\left\langle a^{2}\right\rangle /\left\langle a^{3}\right\rangle=1.5 \times 10^{5} \mathrm{~cm}^{-1}$ before the reverse shock destruction. After the reverse shock destruction, $\left\langle a^{2}\right\rangle /\left\langle a^{3}\right\rangle=4.2 \times 10^{4} \mathrm{~cm}^{-1}$. This is a measure of dust area per dust volume and is also a measure of the $\mathrm{H}_{2}$ formation rate of the dust surface. Small $\left\langle a^{2}\right\rangle /\left\langle a^{3}\right\rangle$ leads to a low $\mathrm{H}_{2}$ formation rate. This is the reason why model A1m9 shows a smaller $\mathrm{H}_{2}$ fraction than $\mathrm{B} 1 \mathrm{~m} 9$ and $\mathrm{C} 1 \mathrm{~m} 9$. The dust destruction by reverse shocks changes not only the dust mass but also the grain size distribution, and as a result drastically suppresses star formation in the galaxy.

In Figure 8, we show the evolution of the $\mathrm{H}_{2}$ formation rate in gas phase, $\left[d f_{\mathrm{H}_{2}} / d t\right]_{\text {gas }}$; the $\mathrm{H}_{2}$ formation rate on dust grain, $\left[d f_{\mathrm{H}_{2}} / d t\right]_{\text {dust }}$; the $\mathrm{H}_{2}$ destruction rate by $\mathrm{UV}$ photons, $\left[d f_{\mathrm{H}_{2}} / d t\right]_{\mathrm{UV}}$; and the $\mathrm{H}_{2}$ decreasing rate by the star formation, $\left[d f_{\mathrm{H}_{2}} / d t\right]_{\text {star }}$, normalized to the total formation rate, $\left[d f_{\mathrm{H}_{2}} / d t\right]_{\mathrm{gas}}+\left[d f_{\mathrm{H}_{2}} / d t\right]_{\text {dust }}$, in model A1m9. At $t \sim 4 \times 10^{7} \mathrm{yr}$, the $\mathrm{H}_{2}$ formation rate on dust grains exceeds the rate in the gas phase. However, the $\mathrm{H}_{2}$ formation rate on dust grains is



Figure 9. Time evolution of SFR in units of $M_{\odot} \mathrm{yr}^{-1}$ in the model with dust destruction for various $n_{\mathrm{SN}}=0.03,0.1,0.3,1.0,3.0$, and $10 \mathrm{~cm}^{-3}$. The values of $n_{\mathrm{SN}}$ are the same as those in Figure 1. The model without dust destruction by the reverse shocks is also shown. The horizontal axis shows the age of the galaxy in units of $\mathrm{yr}$ from the formation redshift, $z_{\mathrm{vir}}=10$.

(A color version of this figure is available in the online journal.)

less than the $\mathrm{H}_{2}$ decreasing rate by star formation at this epoch. At $t \sim 1.6 \times 10^{8} \mathrm{yr}$, the $\mathrm{H}_{2}$ formation rate on dust grains exceeds the $\mathrm{H}_{2}$ decreasing rate by star formation. The formation on dust grains becomes the dominant process among all of $\mathrm{H}_{2}$ formation and destruction processes. From this time when $\sigma_{d,-20} \gtrsim 5.2 \times 10^{-5}$, molecular fraction, $f_{\mathrm{H}_{2}}$, starts to increase. The molecular destruction rate by UV photons does not exceed the molecular decreasing rate by the star formation after $t \sim 4 \times 10^{7}$ yr. After $t \sim 4 \times 10^{7} \mathrm{yr}$, $I_{\mathrm{UV}}\left(3.1 \times 10^{15} \mathrm{~Hz}\right) \sim 2.0-4.8 \times 10^{-20} \mathrm{erg} \mathrm{s}^{-1} \mathrm{~cm}^{-2} \mathrm{~Hz}^{-1} \mathrm{ster}^{-1}$, which corresponds to $J_{21}=20-40$ where $J_{21}$ is in units of $I_{\mathrm{UV}}\left(3.1 \times 10^{15} \mathrm{~Hz}\right)=J_{21} \times 10^{-21} \mathrm{erg} \mathrm{s}^{-1} \mathrm{~cm}^{-2} \mathrm{~Hz}^{-1} \mathrm{ster}^{-1}$. Note that $J_{21}$ in this model is higher than the values, $J_{21} \sim 1$, for the Lyman-Werner background in the redshift of $5<z<10$ suggested in recent papers (e.g., Greif \& Bromm 2006). The destruction process in the gas phase does not affect the evolution of molecular fraction significantly after $t \gtrsim 1 \times 10^{7} \mathrm{yr}$.

\subsection{The ISM Density around SN}

The dependence of the time evolution of SFR on the ISM density around an $\mathrm{SN}$ is presented in Figure 9. We can see that after $t \sim 5 \times 10^{7} \mathrm{yr}$ higher density around SN progenitors results in lower molecular fraction and hence lower star formation efficiency. The SFR, $\Psi(t)$, is independent of $n_{\mathrm{SN}}$ before $t \sim$ $5 \times 10^{7} \mathrm{yr}$, because $\mathrm{H}_{2}$ forms predominantly in the gas phase. In the model without reverse shock destruction, the SFR increases from $\sim 10^{8} \mathrm{yr}$ and saturates around $5 \times 10^{8} \mathrm{yr}$. This is because the gas is consumed by the star formation. In model A10m9 $\left(n_{\mathrm{SN}}=10 \mathrm{~cm}^{-3}\right)$, SFR is suppressed until $t \sim 0.8 \times 10^{9} \mathrm{yr}$.

We should note that it is probably true that $n_{\mathrm{SN}}<1 \mathrm{~cm}^{-3}$ for Population III stars in the mass range of $20-40 M_{\odot}$, since Population III stars are massive and can photoevaporate the clouds in which they form, and ionized flows evacuate the dense gas around the stars to well below $n_{\mathrm{SN}}=1 \mathrm{~cm}^{-3}$ (Whalen et al. 2004; Kitayama et al. 2004). In this case, consideration of circumstellar densities of $10 \mathrm{~cm}^{-3}$ and greater is not relevant to dust evolution in the SNR. In this paper, we consider $n_{\mathrm{SN}}>5 \mathrm{~cm}^{-3}$ for completeness. If the stars are forming at lower redshift and are enriched, they will have stellar winds that also sweep away circumstellar gas to low densities. 


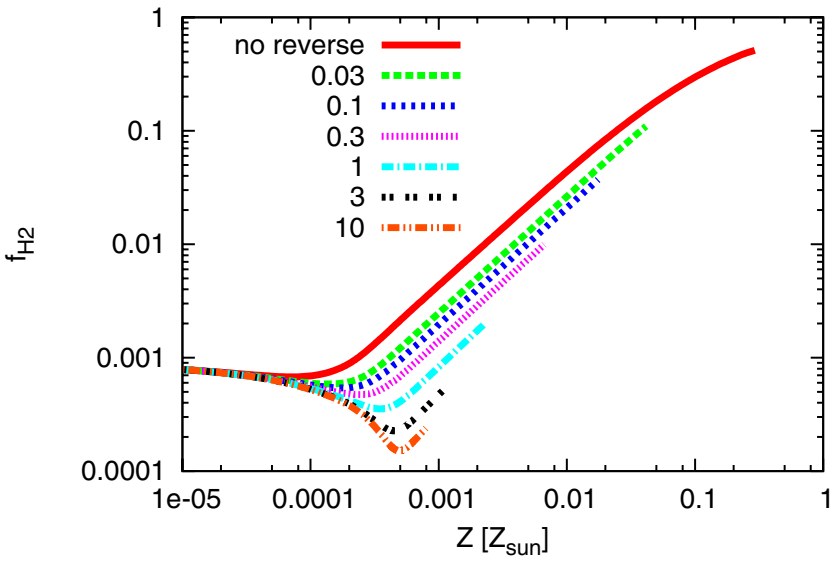

Figure 10. Molecular faction evolution, $f_{\mathrm{H}_{2}}$, for the model of different densities around an SN II, $n_{\mathrm{ISM}, \mathrm{SN}}=0.03,0.1,0.3,1.0,3.0$, and $10 \mathrm{~cm}^{-3}$, which are the same as in Figure 1, and the model without reverse shocks. The horizontal axis shows the metallicity, $Z$, in units of $Z_{\odot}$.

(A color version of this figure is available in the online journal.)

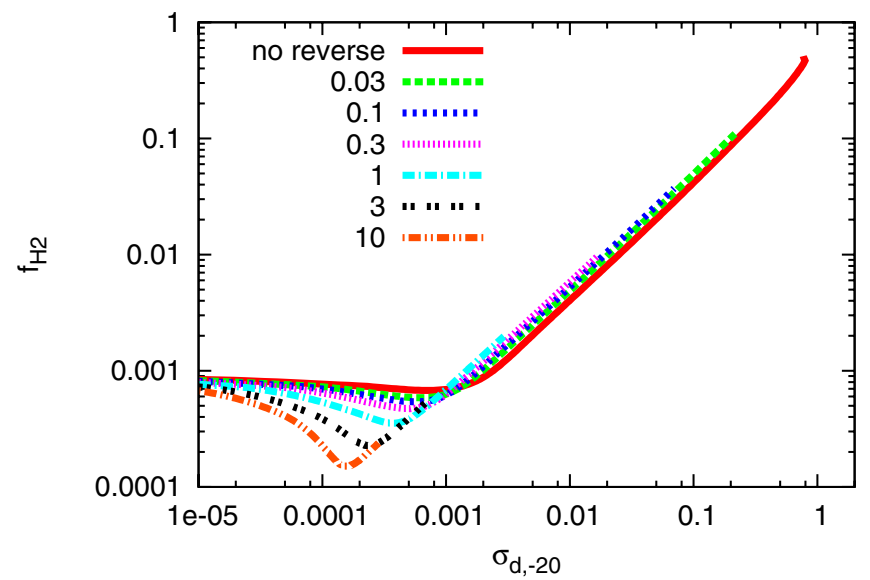

Figure 11. Molecular faction evolution, $f_{\mathrm{H}_{2}}$, for the model of different densities around an SN II, $n_{\mathrm{ISM}, \mathrm{SN}}=0.03,0.1,0.3,1.0,3.0$, and $10 \mathrm{~cm}^{-3}$, which are the same as in Figure 1, and the model without reverse shocks. The horizontal axis shows the total dust cross section per unit volume, $\sigma_{d,-20}$, in units of $10^{-20} \mathrm{~cm}^{-1}$.

(A color version of this figure is available in the online journal.)

We note that in a usual star formation recipe in both numerical simulations and analytic models, the SFR is assumed to increase with the local gas density, and our results show that the SFR is strongly affected by $n_{\mathrm{SN}}$. We will discuss the effects of $n_{\mathrm{SN}}$ on the SFR in more detail in Section 5.

In Figure 10, we show the change of the molecular fraction, $f_{\mathrm{H}_{2}}$, with metallicity, $Z$, for various $n_{\mathrm{SN}}$. In usual chemical evolution models, $Z$ is a key indicator of galaxy evolution. However, as shown in this figure, $f_{\mathrm{H}_{2}}$ does not solely depend on the metallicity. For $Z \gtrsim 5 \times 10^{-4} Z_{\odot}, f_{\mathrm{H}_{2}}$ is large in models with small $n_{\mathrm{SN}}$. This is because $D_{\text {area }} / M_{\text {metal }}$ is large (small) in models with small (large) $n_{\mathrm{SN}}$ for the same $Z$ as shown in Figure 3.

The molecular fraction is well described by the total dust cross section per unit volume for $\sigma_{d,-20} \gtrsim 0.001$. In Figure 11, we show the evolution of the molecular fraction in terms of total dust cross section per unit volume. For $\sigma_{d,-20} \gtrsim 1 \times 10^{-3}, \mathrm{H}_{2}$ formation on dust grains dominates $f_{\mathrm{H}_{2}}$ evolution as shown by the convergence of all models.

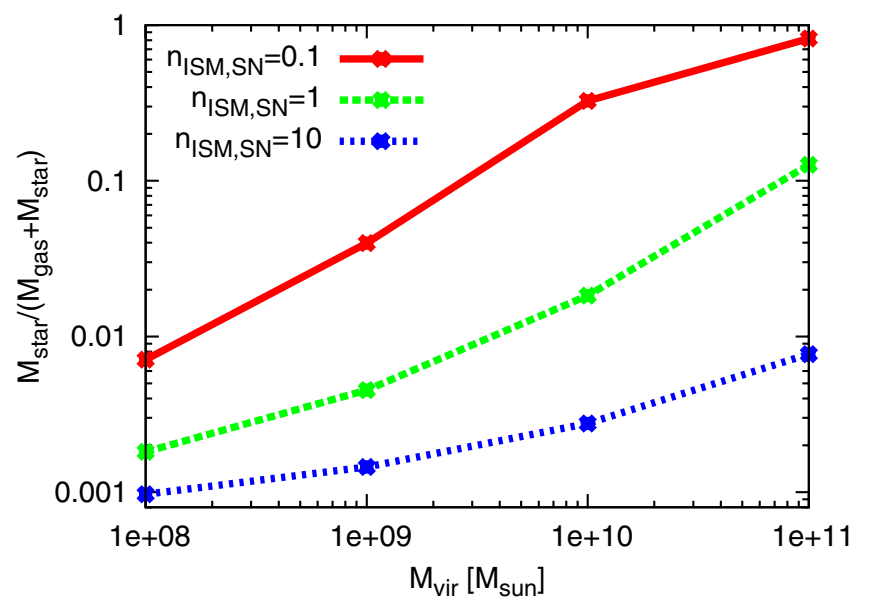

Figure 12. Stellar mass fraction in the galaxy, $M_{\mathrm{star}} /\left(M_{\mathrm{gas}}+M_{\mathrm{star}}\right)$, for the various virial masses, $M_{\mathrm{vir}}$, of the models with $n_{\mathrm{SN}}=0.1 \mathrm{~cm}^{-3}$ (solid line: models A0.1m8, A0.1m9, A0.1m10, and A0.1m11), $n_{\mathrm{ISM} . \mathrm{SN}}=1.0 \mathrm{~cm}^{-3}$ (long dashed line: models A1m8, A1m9, A1m10, and A1m11), and $n_{\mathrm{ISM} . \mathrm{SN}}=$ $10 \mathrm{~cm}^{-3}$ (short dashed line: models A10m8, A10m9, A10m10, and A10m10).

(A color version of this figure is available in the online journal.)

\subsection{The Dark Matter Halo Mass}

Finally, we show the stellar mass fraction, $M_{\text {star }} /\left(M_{\text {gas }}+M_{\text {star }}\right)$ for $M_{\mathrm{vir}}=10^{8}, 10^{9}, 10^{10}$, and $10^{11} M_{\odot}$ at $z_{\mathrm{vir}}=10 \mathrm{in} \mathrm{Figure} 12$. We note that dark halos of virial masses, $M_{\mathrm{vir}}=10^{8}, 10^{9}, 10^{10}$, and $10^{11} M_{\odot}$ correspond to the density fluctuation of $2.0 \sigma$, $2.5 \sigma, 3.0 \sigma$, and $4.1 \sigma$, respectively. $M_{\text {star }} /\left(M_{\text {gas }}+M_{\text {star }}\right)$ is large for large $M_{\mathrm{vir}}$. This is explained as follows: the gas cools to the CMB temperature $\sim 30 \mathrm{~K}$ in all $M_{\text {vir }}$ after $t \sim$ a few $\times 10^{7} \mathrm{yr}$, although $T_{\text {vir }}$ increases with $M_{\text {vir }}$, so that the final gas density becomes higher because of smaller $H / r_{\text {disk }}$ in larger $M_{\text {vir }}$ (see Equation (15)). This results in more rapid molecular formation in a larger $M_{\text {vir }}$ halo (see Equation (23)). The rapid molecular formation enhances the star formation and as a result causes the large stellar mass fraction. We should note that in higher $z_{\text {vir }}$, $M_{\text {star }} /\left(M_{\text {gas }}+M_{\text {star }}\right)$ is larger for the galaxies with $T_{\text {vir }} \gtrsim 10^{4} \mathrm{~K}$, since the rotation timescale becomes smaller in our model.

\section{DISCUSSION AND CONCLUSIONS}

We have investigated the dust size evolution and the resulting $\mathrm{H}_{2}$ formation on dust grains in the galaxies in the early universe $(z>5)$. Our dust evolution model includes the dust production by SNe II and the effects of dust destruction by the reverse and forward shocks driven by $\mathrm{SNe}$. In the galaxy model, we follow the chemical network of $\mathrm{H}_{2}$ formation and the thermal evolution of gas, taking into account cooling by $\mathrm{H}_{2}$, atomic hydrogen and metals, and heating by the stellar radiation. The SFR is assumed to be proportional to the mass of molecular hydrogen and the rotation timescale of the galactic gas disk. The evolution of the size distribution of dust has been taken into account for the first time in this paper to investigate the influence on star formation activity in galaxies.

We obtain three main results. First, we show that $\mathrm{H}_{2}$ formation is suppressed by dust destruction, especially that resulting from the reverse shocks in SNRs. The resulting molecular fraction in the galaxy model with dust destruction by both reverse shocks and forward shocks (see Figure 7) is 2.5 orders of magnitude less than the galaxy model without both destruction processes (see Figure 5) and is two orders less than the galaxy model considering dust destruction by only forward shocks (see 
Figure 6) for $n_{\mathrm{SN}}=1.0 \mathrm{~cm}^{-3}$. Our results show that dust size evolution has great effects on the early galaxy evolution.

The dust destruction by reverse shocks is essential in our galaxy model and has a more dominant role than forward shocks, since the reverse shock destroys small dust grains earlier than the forward shock. Dust destruction by a reverse shock becomes more (less) prominent for a higher (lower) $n_{\mathrm{SN}}$. In early galaxy evolution, considering dust destruction by reverse shocks is very important to $\mathrm{H}_{2}$ formation. We note that forward shocks affect dust size distribution in the large dust-to-gas mass ratio, $\mathcal{D} \gtrsim 10^{-3}$, as shown in Section 4.1 .

Second, we show that the SFR strongly depends on the ISM density around $\mathrm{SN}$ progenitors, $n_{\mathrm{SN}}$, which determines the efficiency of dust destruction by reverse shocks. As noted in Section 3 , we treat $n_{\mathrm{SN}}$ differently from the mean gas density in our one-zone galaxy model, $n_{\mathrm{H}}$, taking into account the effect of photoionization by $\mathrm{SN}$ progenitors. The molecular fraction is different among the models with various $n_{\mathrm{SN}}$, even for the same $Z$ (see Figure 10), and it solely depends on the total dust cross section per volume from the time when $\mathrm{H}_{2}$ formation on dust grains becomes the most dominant of the processes of $\mathrm{H}_{2}$ formation and destruction (see Figure 11). The ratio of the total dust cross section to total metal mass presented in Figure 3 is very useful for a numerical simulation of galaxy formation with dust size evolution.

A higher ISM density around $\mathrm{SN}$ progenitors, $n_{\mathrm{SN}}$, results in a lower SFR in the early galaxy evolution. In conventional galaxy formation theory, SFR has been assumed as an increasing function of gas density. Since in our one-zone model we simply treat $n_{\mathrm{SN}}$ as a parameter, it is very interesting to resolve the ionized region around an SN progenitor by high-resolution radiation hydrodynamic simulation taking into account the effects of ionization heating by massive SN progenitors.

Finally, we show that galaxy evolution depends on $M_{\mathrm{vir}}$ and that the stellar mass fraction, $M_{\mathrm{star}} /\left(M_{\mathrm{gas}}+M_{\mathrm{star}}\right)$, is monotonically increasing functions of $M_{\mathrm{vir}}$. The halo number density in the redshift range $5<z<10$ is a decreasing function of the halo virial mass, $10^{8} M_{\odot}<M_{\text {vir }}<10^{11} M_{\odot}$. In most papers on the reionization, $M_{\text {star }} /\left(M_{\text {gas }}+M_{\text {star }}\right)$ is assumed to be constant for various halo masses, $M_{\mathrm{vir}}$. The difference of stellar mass fraction with different $M_{\text {vir }}$ in our results is important for galaxy evolution in the early universe and should be considered in the cosmic reionization process. We will study this effect taking into account Population III star formation and the $\mathrm{H}_{2}$ dissociation by the Lyman-Werner background in a forthcoming paper, since a strong Lyman-Werner background that is effective for dissociating molecular hydrogen, delaying gas condensation, and suppressing star formation especially in the lower mass $\mathrm{H}_{2}$ cooling halos is expected in the cosmic reionization era (D. Yamasawa et al. 2011, in preparation).

Dust size distribution governs the extinction curve and reemitting IR spectrum (e.g., Silva et al. 1998; Granato et al. 2000; Takeuchi et al. 2005; Li et al. 2008). A ground-based interferometric facility, the Atacama Large Millimeter Array (ALMA), ${ }^{5}$ can be used to study high-redshift galaxies, since redshifted dust emission can be observed with ALMA. We can study dust emission and the resulting SED of our galaxy model using our results of the dust size distribution and test our galaxy model by comparing it to observational data in the future.

Our galaxy model can be applied to the lower redshift $(z<5)$ star formation history, taking into account dust production by

\footnotetext{
5 http://www.almaobservatory.org/
}

low-mass stars and growth of dust due to accretion of metal in ISM (Inoue 2003; Draine 2009). The accretion timescale, $\tau_{\text {acc }}$, depends on the metallicity and is given by $\tau_{\text {acc }}=\tau_{\text {acc }, 0} Z_{\odot} / Z$, where $\tau_{\text {acc }, 0} \sim 10^{8} \mathrm{yr}$ (Inoue 2003). If we assume $Z \sim 10^{-2} Z_{\odot}$ corresponding $\tau_{\text {acc }} \sim 10^{10} \mathrm{yr}$, then the accretion time is too long to affect dust size distribution for a cosmic time $\leqslant 1.2 \mathrm{Gyr}$ $(z>5)$. At a galaxy age of $\gtrsim 1 \mathrm{Gyr}$, dust production by lowmass stars should affect the dust size evolution, because after that epoch dust is supplied from low-mass stars as well as $\mathrm{SNe}$ II. We will include such processes to investigate low-redshift galaxies in the future work.

Cosmological simulation of galaxy formation including our dust formation and evolution model is needed, since it is widely understood that most $z \sim 10$ galaxies were not clear disk galaxies (Johnson et al. 2008; Greif et al. 2010; Wise et al. 2010). We will study first galaxy formation by cosmological simulation including our dust model.

We thank the anonymous referee for the very careful reading and comments that improved this paper. The authors are grateful to M. Fujimoto, K. Sorai, K. Omukai, A. K. Inoue, T. Takeuchi, N. Yoshida, and B. T. Draine for helpful discussions. Numerical computations were carried out on NEC SX-9 at the Center for Computational Astrophysics, CfCA, of National Astronomical Observatory of Japan. This work was partly supported by the Grant-in-Aid for Scientific Research of Japan Society for the Promotion of Sciences (08091823, 18104003, 0340038). H.H. is supported by NSC grant 99-2112-M-001-006-MY3. T.N. and K.N. are supported by the World Premier International Research Center Initiative, MEXT, Japan.

\section{REFERENCES}

Abel, T., Anninos, P., Zhang, Y., \& Norman, M. L. 1997, New Astron., 2, 181 Barlow, M. J., et al. 2010, A\&A, 518, 138 Bianchi, S., \& Schneider, R. 2007, MNRAS, 378, 973

Bigiel, F., Leroy, A., Walter, F., Brinks, E., Blok, W. J. G., Madore, B., \& Thornley, M. D. 2008, AJ, 136, 2846

Bromm, V., Yoshida, N., \& Hernquist, L. 2003, ApJ, 596, 135

Bromm, V., Yoshida, N., Hernquist, L., \& McKee, C. F. 2009, Nature, 459, 49 Cazaux, S., \& Spaans, M. 2004, ApJ, 611, 40

Cherchneff, I., \& Dwek, E. 2010, ApJ, 713, 1

Cole, S., Lacey, C. G., Baugh, C. M., \& Frenk, C. S. 2000, MNRAS, 319, 168 Cox, A. N. 2000, Allen's Astrophysical Quantities (4th ed.; New York: Springer) Draine, B. T. 2009, in ASP Conf. Ser. 414, Cosmic Dust-Near and Far, ed. Th.

Henning, E. Grün, \& J. Steinacker (San Francisco, CA: ASP), 453

Draine, B. T., \& Bertoldi, F. 1996, ApJ, 468, 269

Draine, B. T., \& Lee, H. M. 1984, ApJ, 285, 89

Dwek, E., \& Cherchneff, I. 2011, ApJ, 727, 63

Dwek, E., Galliano, F., \& Jones, A. P. 2007, ApJ, 662, 927

Ferrara, A., Pettoni, M., \& Sjcjekinov, Y. 2000, MNRAS, 319, 539

Ferrarotti, A. S., \& Gail, H.-P. 2006, A\&A, 447, 553

Gall, C., Andersen, A. C., \& Hjorth, J. 2011, A\&A, 528, A13

Gallerani, S., et al. 2010, arXiv: 1006.4463

Galli, D., \& Palla, F. 1998, A\&A, 335, 403

Glover, S. C. O., \& Abel, T. 2008, MNRAS, 388, 1627

Glover, S. C. O., \& Jappsen, A.-K. 2007, ApJ, 666, 1

Gnedin, N. Y., Tassis, K., \& Kravtsov, A. V. 2009, ApJ, 697, 55

Granato, G. L., Lacey, C. G., Silva, L., Bressan, A., Baugh, C. M., Cole, S., \& Frenk, C. S. 2000, ApJ, 542, 710

Greif, T. H., \& Bromm, V. 2006, MNRAS, 373, 128

Greif, T. H., Glover, S. C. O., Bromm, V., \& Klessen, R. 2010, ApJ, 716, 510 Haiman, Z., Thoul, A. A., \& Loeb, A. 1996, ApJ, 464, 523

Heger, A., Fryer, C. L., Woosley, S. E., Langer, N., \& Hartmann, D. H. 2003, ApJ, 591,288

Heger, A., \& Woosley, S. E. 2002, ApJ, 567, 532

Hirashita, H., \& Ferrara, A. 2002, MNRAS, 337, 921

Hollenbach, D., \& McKee, C. F. 1989, ApJ, 342, 306

Hutchings, R. M., Santoro, F., Thomas, P. A., \& Couchman, H. M. P. 2002, MNRAS, 330, 927 
Inoue, A. K. 2003, PASJ, 55, 901

Jappsen, A.-K., Glover, S. C. O., Klessen, R. S., \& Mac Low, M.-M. 2007, ApJ, 660, 1332

Jappsen, A.-K., Klessen, R. S., Glover, S. C. O., \& Mac Low, M.-M. 2009a, ApJ, 696, 1065

Jappsen, A.-K., Mac Low, M.-M., Glover, S. C. O., \& Klessen, R. S. 2009b, ApJ, 694, 1161

Joggerst, C. C., Akmgren, A., Bell, J., Heger, A., Whalen, D., \& Woosley, S. E. 2010a, ApJ, 709, 11

Joggerst, C. C., Akmgren, A., \& Woosley, S. E. 2010b, ApJ, 723, 353

Johnson, J. L., Greif, T. H., \& Bromm, V. 2008, MNRAS, 388, 26

Jones, A. P., Tielens, A. G. G. M., Hollenbach, D. J., \& McKee, C. F. 1994, ApJ, 433, 797

Kennicutt, R. C. 1998, ApJ, 498, 541

Kitayama, T., \& Ikeuchi, S. 2000, ApJ, 529, 615

Kitayama, T., \& Yoshida, N. 2005, ApJ, 630, 675

Kitayama, T., Yoshida, N., Susa, H., \& Umemura, M. 2004, ApJ, 613, 631

Kozasa, T., Nozawa, T., Tominaga, N., Umeda, H., Maeda, K., \& Nomoto, K. 2009, in ASP Conf. Ser. 414, Cosmic Dust-Near and Far, ed. Th. Henning, E. Grün, \& J. Steinacker (San Francisco, CA: ASP), 43

Krügel, E. 2008, An Introduction to the Physics of Interstellar Dust (London: Taylor and Francis)

Krumholz, M. R., \& McKee, C. F. 2005, ApJ, 630, 250

Li, Y., Hopkins, P. F., Hernquist, L., Finkbeiner, D. P., Cox, T. J., Springel, V., Jiang, L., \& Yoshida, N. 2008, ApJ, 678, 41

Machacek, M. E., Bryan, G. L., \& Abel, T. 2001, ApJ, 548, 509

Machacek, M. E., Bryan, G. L., \& Abel, T. 2003, MNRAS, 338, 273

Mackey, J., Bromm, V., \& Hernquist, L. 2003, ApJ, 586, 1

Maiolino, R., Schneider, R., Oliva, E., Bianchi, S., Ferrara, A., Mannucci, F., Pedani, M., \& Roca Sogorb, M. 2004, Nature, 431, 533

Mathis, J. S., Rumpl, W., \& Nordsieck, K. H. 1977, ApJ, 217, 425

Mo, H. J., Mao, S., \& White, S. D. M 1998, MNRAS, 295, 319

Nath, B. B., Laskar, T., \& Shull, J. M. 2008, ApJ, 682, 1055

Navarro, J. F., Frenk, C. S., \& White, S. D. M. 1996, ApJ, 462, 563

Nozawa, T., Kozasa, T., \& Habe, A. 2006, ApJ, 648, 435

Nozawa, T., Kozasa, T., Habe, A., Dwek, E., Umeda, H., Tominaga, N., Maeda, K., \& Nomoto, K. 2007, ApJ, 666, 955

Nozawa, T., Kozasa, T., Tominaga, N., Maeda, K., Umeda, H., Nomoto, K., \& Oliver, K. 2010, ApJ, 713, 356

Nozawa, T., Kozasa, T., Umeda, H., Maeda, K., \& Nomoto, K. 2003, ApJ, 598, 785

Nozawa, T., Maeda, K., Kozasa, T., Tanaka, M., Nomoto, K., \& Umeda, H. 2011, arXiv:1105.0973
Omukai, K. 2000, ApJ, 534, 809

Omukai, K., Hosokawa, T., \& Yoshida, N. 2010, ApJ, 722, 1793

Omukai, K., Tsuribe, T., Schneider, R., \& Ferrara, A. 2005, ApJ, 626, 627

O'Shea, B. W., \& Norman, M. L. 2008, ApJ, 673, 14

Pollack, L. B., Hollenbach, D., Beckwith, S., Simonelli, D. P., Roush, T., \& Fong, W. 1994, ApJ, 421, 615

Robertson, B. E., \& Kravtsov, A. V. 2008, ApJ, 680, 1083

Salpeter, E. E. 1955, ApJ, 121, 161

Schaerer, D. 2002, A\&A, 382, 28

Schneider, R., Ferrara, A., \& Salvaterra, R. 2004, MNRAS, 351, 1379

Schneider, R., \& Omukai, K. 2010, MNRAS, 402, 429

Schneider, R., Omukai, K., Inoue, A., \& Ferrara, A. 2006, MNRAS, 369, 1437

Shakura, N. I., \& Sunyaev, R. A. 1988, Adv. Space Res., 8, 135

Sibthorpe, B., et al. 2010, ApJ, 719, 1553

Silva, L., Granato, G. L., Bressan, A., \& Danese, L. 1998, ApJ, 509, 103

Silvia, D. W., Smith, B. D., \& Shull, J. M. 2010, ApJ, 715, 1575

Smith, B., Sigurdsson, S., \& Abel, T. 2008, MNRAS, 385, 1443

Smith, B. D., Turk, M. J., Sigurdsson, S., O’shea, B. W., \& Norman, M. L. 2009, ApJ, 691, 441

Spergel, D. N., et al. 2007, ApJS, 177, 377

Springel, V., et al. 2005, Nature, 435, 629

Susa, H. 2007, ApJ, 659, 908

Susa, H. 2008, ApJ, 684, 226

Takeuchi, T., Ishii, T. T., Nozawa, T., Kozasa, T., \& Hirashita, H. 2005, MNRAS, 362,592

Tegmark, M., Silk, J., Rees, M. J., Blanchard, A., \& Palla, F. 1997, ApJ, 474, 1

Todini, P., \& Ferrara, A. 2001, MNRAS, 325, 726

Umeda, H., \& Nomoto, K. 2002, ApJ, 565, 385

Valiante, R., Schneider, R., Bianchi, S., \& Andersen, A. C. 2009, MNRAS, 397, 1661

Whalen, D., Abel, T., \& Norman, M. L. 2004, ApJ, 610, 14

Whalen, D., van Veelen, B., O'shea, B. W., \& Norman, M. L. 2008, ApJ, 682, 49

Wise, J. H., \& Abel, T. 2007, ApJ, 671, 1559

Wise, J. H., \& Abel, T. 2008, ApJ, 685, 40

Wise, J. H., \& Cen, R. 2009, ApJ, 693, 984

Wise, J. H., Turk, M. J., Norman, M. L., \& Abel, T. 2010, arXiv:1011.2632v2

Wolfe, A. M., \& Chen, H.-W. 2006, ApJ, 652, 981

Yoshida, N., Abel, T., Hernquist, L., \& Sugiyama, N. 2003, ApJ, 592, 645

Yoshida, N., Omukai, K., \& Hernquist, L. 2008, Science, 321, 669

Zhukovska, S., Gail, H.-P., \& Trieloff, M. 2008, A\&A, 479, 453 